\title{
A Fourier restriction theorem for a perturbed hyperbolic paraboloid: polynomial partitioning
}

\author{
Stefan Buschenhenke ${ }^{1}$. Detlef Müller ${ }^{1} \cdot$ Ana Vargas $^{2}$
}

Received: 17 November 2020 / Accepted: 23 November 2021 / Published online: 7 February 2022

(c) The Author(s) 2022

\begin{abstract}
We consider a surface with negative curvature in $\mathbb{R}^{3}$, which is a cubic perturbation of the saddle. For this surface, we prove a new restriction theorem, analogous to the theorem for paraboloids proved by L. Guth in 2016. This specific perturbation has turned out to be of fundamental importance also to the understanding of more general classes of one-variate perturbations, and we hope that the present paper will further help to pave the way for the study of general perturbations of the saddle by means of the polynomial partitioning method.
\end{abstract}

Keywords Hyperbolic hypersurface $\cdot$ Fourier restriction · Polynomial partitioning

Mathematics Subject Classification 42B25

\section{Contents}

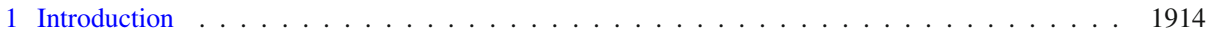

2 Broad points . . . . . . . . . . . . . . . . . . . . . . . . . . 1916

3 Reduction of Theorem 2.1 to a setup allowing for inductive arguments . . . . . . . . . . . . . 1918

4 Proof of Theorem $3.2 \ldots \ldots \ldots$. . . . . . . . . . . . . . . . . . . . . 1920

Stefan Buschenhenke was partially supported by the ERC grant 307617. Stefan Buschenhenke and Detlef Müller were partially supported by the DFG Grants MU 761/ 11-1 and MU 761/ 11-2. Ana Vargas was partially supported by Grants PID2019-105599GB-I00 / AEI / 10.13039/501100011033 (Ministerio de Ciencia e innovación) and MTM2016-76566-P (Ministerio de Ciencia, Innovación y Universidades), Spain.

$凶 \quad$ Detlef Müller

mueller@math.uni-kiel.de

http://www.math.uni-kiel.de/analysis/de/mueller

Stefan Buschenhenke

buschenhenke@math.uni-kiel.de

http://www.math.uni-kiel.de/analysis/de/buschenhenke

Ana Vargas

ana.vargas@uam.es

http://matematicas.uam.es/ AFA/

1 Mathematisches Seminar, C. A.-Universität Kiel, Heinrich-Hecht-Platz 6, 24118 Kiel, Germany

2 Departmento de Mathemáticas, Universidad Autónoma de Madrid, 28049 Madrid, Spain 
4.1 Transversality for bilinear estimates . . . . . . . . . . . . . . . . . . . . . . . . 1923

4.2 Completing the proof of Theorem $3.2 \ldots \ldots \ldots$. . . . . . . . . . . . . . . 1931

4.3 Proof of the Geometric Lemma . . . . . . . . . . . . . . . . . . . . . . . . . . . . . . . . 1935

5 Passing from extension estimates on cubes to estimates on plates: an orthogonality lemma . . . 1936 References . . . . . . . . . . . . . . . . . . . . . . 1937

\section{Introduction}

Let $S \subset \mathbb{R}^{n}$ be a smooth hypersurface. The Fourier restriction problem, introduced by E. M. Stein in the seventies (for general submanifolds), asks for the range of exponents $\tilde{p}$ and $\tilde{q}$ for which an a priori estimate of the form

$$
\left(\int_{S}|\widehat{f}|^{\tilde{q}} d \sigma\right)^{1 / \tilde{q}} \leq C\|f\|_{L^{\tilde{p}}\left(\mathbb{R}^{n}\right)}
$$

holds true for every Schwartz function $f \in \mathcal{S}\left(\mathbb{R}^{n}\right)$, with a constant $C$ independent of $f$. Here, $d \sigma$ denotes the surface measure on $S$.

The sharp range in dimension $n=2$ for curves with non-vanishing curvature was determined through work by Fefferman et al. [13,38]. In higher dimension, the sharp $L^{\tilde{p}}-L^{2}$ result for hypersurfaces with non-vanishing Gaussian curvature was obtained by Stein and Tomas [27,34] (see also Strichartz [29]). Some more general classes of surfaces were treated by Greenleaf [16]. In work by Ikromov, Kempe and Müller [18] and Ikromov and Müller [19,20], the sharp range of Stein-Tomas type $L^{\tilde{p}}-L^{2}$ restriction estimates has been determined for a large class of smooth, finite-type hypersurfaces, including all analytic hypersurfaces.

The question about general $L^{\tilde{p}}-L^{\tilde{q}}$ restriction estimates is nevertheless still wide open. Fourier restriction to hypersurfaces with non-negative principal curvatures has been studied intensively by many authors. Major progress was due to J. Bourgain in the nineties [4-6]. At the end of that decade the bilinear method was introduced [23-25,30-33,37]. A new impulse to the problem has been given with the multilinear method [3,7]. The best results up to date have been obtained with the polynomial partitioning method, developed by Guth $[14,15]$ (see also $[17,36]$ for recent improvements).

For the case of hypersurfaces of non-vanishing Gaussian curvature but principal curvatures of different signs, besides Tomas-Stein type Fourier restriction estimates, until recently the only case which had been studied successfully was the case of the hyperbolic paraboloid (or "saddle") in $\mathbb{R}^{3}$ : in 2015, independently Lee [22] and Vargas [35] established results analogous to Tao's theorem [30] on elliptic surfaces (such as the 2 -sphere), with the exception of the end-point, by means of the bilinear method. Recently, Stovall [28] was able to include also the end-point case. Moreover, Cho and Lee [12], and Kim [21], improved the range by adapting ideas by Guth $[14,15]$ which are based on the polynomial partitioning method. Results on higher dimensional hyperbolic paraboloids have just been reported by Barron [1].

In our previous paper [9], we considered a one variable perturbation of the hyperbolic paraboloid, and applied the bilinear method, obtaining results analogous to [22,35]. Further results for more general classes of one-variate finite type, respectively flat, perturbations based on the bilinear method were obtained in $[10,11]$. Bilinear estimates are also key elements in the results obtained with the polynomial partitioning method for the non-negative curvature case. With the base of our previous bilinear results, we explore in this article the application of that method to our model surfaces. We obtain the analogous result to [14] for our class of hyperbolic surfaces. 
More precisely, we consider the family of functions

$$
\phi_{\gamma}(x, y)=x y+\frac{\gamma}{3} y^{3} \quad \text { for }-1 \leq \gamma \leq 1,
$$

defined on $\Sigma:=[0,1] \times[0,1]$, and the corresponding surfaces

$$
S_{\gamma}=\left\{\left(x, y, \phi_{\gamma}(x, y)\right):(x, y) \in \Sigma\right\} .
$$

The associated adjoints to the corresponding Fourier restriction operators are the extension operators given by

$$
\mathcal{E}_{\gamma}(\xi):=\int_{\Sigma} f(x, y) e^{i\left[\xi_{1} x+\xi_{2} y+\xi_{3} \phi_{\gamma}(x, y)\right]} d x d y, \quad \xi=\left(\xi_{1}, \xi_{2}, \xi_{3}\right) \in \mathbb{R}^{3} .
$$

Our main result will be the following analogue of the results by Bassam Shayya for elliptic surfaces with weighted norms [26] and by Jongchon Kim for the unperturbed hyperbolic paraboloid [21]:

Theorem 1.1 For any $p>3.25$ with $p>2 q^{\prime}$, there is a constant $C_{p, q}$ which is independent of $\gamma \in[-1,1]$ such that

$$
\left\|\mathcal{E}_{\gamma} f\right\|_{L^{p}\left(\mathbb{R}^{3}\right)} \leq C_{p, q}\|f\|_{L^{q}(\Sigma)}
$$

for all $f \in L^{q}(\Sigma)$.

Remark 1.2 (i) Note that in this result and the corresponding Fourier restriction estimate we can replace the domain $\Sigma:=[0,1] \times[0,1]$ by the larger neighborhood $[-1,1] \times[-1,1]$ of the origin, simply by dividing the latter into four sectors of angle $\pi / 2$ and reducing the corresponding estimates in each of these sectors to the estimate given in the theorem by means of symmetry considerations.

ii) Our arguments in this paper easily extend to more general perturbations of $x y$ of cubic type in the sense of [10] in place of the perturbation $\frac{\gamma}{3} y^{3}$, and the same reasoning as in [10] then allows to prove Fourier restriction to surfaces given as the graph of $\phi(x, y):=x y+h(y)$, where the function $h$ is smooth and of finite type at the origin, in the same range of $p$ 's and $q$ 's as in Theorem 1.1.

To simplify the understanding of this paper, we will closely follow the notation and structure of the paper [14], which makes use of induction on scales arguments.

Denote by $B_{R}$ the cube $B_{R}:=[-R, R]^{3}, R \geq 0$. For technical reasons that will become clear soon we shall not be able to induct on an $L^{\infty} \rightarrow L^{3.25}$ estimate for $\mathcal{E}_{\gamma}$ as in [14] (Theorem 2.2). Instead, we shall induct on the following statement:

Theorem 1.3 For any $\epsilon>0$, there is a constant $C_{\epsilon}$ such that for any $\gamma \in[-1,1]$ and for any $R \geq 1$

$$
\left\|\mathcal{E}_{\gamma} f\right\|_{L^{3.25}\left(B_{R}\right)} \leq C_{\epsilon} R^{\epsilon}\|f\|_{L^{2}(\Sigma)}^{2 / q}\|f\|_{L^{\infty}(\Sigma)}^{1-2 / q},
$$

for all $3.25 \geq q>2.6$ and all $f \in L^{\infty}(\Sigma)$.

Applying this estimate to characteristic functions, we obtain the estimate

$$
\left\|\mathcal{E}_{\gamma} f\right\|_{L^{3.25}\left(B_{R}\right)} \leq C_{\epsilon} R^{\epsilon}\|f\|_{L^{q, 1}(\Sigma)},
$$

for all $q>2.6$. Real interpolation with the trivial $L^{1} \rightarrow L^{\infty}$ estimate for the extension operator then gives

$$
\left\|\mathcal{E}_{\gamma} f\right\|_{L^{p}\left(B_{R}\right)} \leq C_{\epsilon} R^{\epsilon}\|f\|_{L^{q}(\Sigma)}
$$


for all $p>3.25, p>2 q^{\prime}$. Finally, an $\epsilon$-removal theorem (Theorem 5.3 in [21]) gives Theorem 1.1.

\section{Broad points}

Definition 2.1 Fix $K \gg 1$ to be a large dyadic number. We introduce four different partitions of the square $\Sigma=[0,1] \times[0,1]$ :

We divide $\Sigma$ into $K^{2}$ disjoint squares (called caps) $\tau$ of sidelength $K^{-1}$. For a cap $\tau$, we denote by $f_{\tau}:=f \chi_{\tau}$. This basic decomposition into caps will play a fundamental role in many places of our subsequent arguments, as in [14]. However, in contrast to [14], it will play no role in the definition of $\alpha$-broadness given below. For the latter notion, the next three decompositions will be relevant:

We divide $\Sigma$ into $K^{1 / 4}$ disjoint long horizontal strips $L$ of dimensions $1 \times K^{-1 / 4}$, we divide $\Sigma$ into $K^{1 / 2}$ disjoint long vertical strips $L$ of dimensions $K^{-1 / 2} \times 1$ and, finally, we divide $\Sigma$ into $K^{3 / 4}$ disjoint short vertical strips $L$ of dimensions $K^{-1 / 2} \times K^{-1 / 4}$, by looking at all intersections of a long horizontal with a long vertical strip. For a strip $L$, we denote by $f_{L}:=f \chi_{L}$.

Let $\alpha \in(0,1)$. Given the function $f, \gamma \in[-1,1]$ and $K$, we say that the point $\xi \in \mathbb{R}^{3}$ is $\alpha$-broad for $\mathcal{E}_{\gamma} f$ if

$$
\max _{L}\left|\mathcal{E}_{\gamma} f_{L}(\xi)\right| \leq \alpha\left|\mathcal{E}_{\gamma} f(\xi)\right|,
$$

where the $\max _{L}$ is taken over all

(a) horizontal strips as above if $|\gamma| K^{1 / 2} \geq 1$, or

(b) horizontal and vertical strips as above if $|\gamma| K^{1 / 2}<1$.

We define $B r_{\alpha} \mathcal{E}_{\gamma} f(\xi)$ to be $\left|\mathcal{E}_{\gamma} f(\xi)\right|$ if $\xi$ is $\alpha$-broad, and zero otherwise.

Note: In contrast to [14], we shall here consider the functions $f$ to be defined on the square $\Sigma$, which will have slight technical advantages, whereas Guth views them as functions on the surface $S_{\gamma}$. Of course, we can as well identify our functions $f$ with the corresponding functions $\left(x, y, \phi_{\gamma}(x, y)\right) \mapsto f(x, y)$ on $S_{\gamma}$. Accordingly, one can identify our "caps" $\tau$ and strips $L$ with the corresponding subsets of the surface $S_{\gamma}$ that are the graphs of $\phi_{\gamma}$ over these sets. This explain why we still like to call the sets $\tau$ "caps".

We will prove the following analogue to Theorem 2.4. in [14]:

Theorem 2.1 For any $0<\epsilon<10^{-10}$, there are constants $K=K(\epsilon) \gg 1$ and $C_{\epsilon}$ such that for any radius $R \geq 1$ and for any $|\gamma| \leq 1$

$$
\left\|B r_{K^{-\epsilon}} \mathcal{E}_{\gamma} f\right\|_{L^{3.25}\left(B_{R}\right)} \leq C_{\epsilon} R^{\epsilon}\|f\|_{L^{2}(\Sigma)}^{12 / 13}\|f\|_{L^{\infty}(\Sigma)}^{1 / 13}
$$

for all $f \in L^{\infty}(\Sigma)$. Moreover $K(\epsilon) \rightarrow \infty$ as $\epsilon \rightarrow 0$.

Note that Theorem 1.3 follows from this theorem by arguments that are similar to those in [14]. To show this, let us put $p:=3.25$.

We divide the domain of integration $B_{R}$ in (1.3) into four subsets:

$A:=\left\{\xi \in B_{R}: \xi\right.$ is $K^{-\epsilon}-$ broad for $\left.\mathcal{E}_{\gamma} f\right\}$,

$B:=\left\{\xi \in B_{R}:\left|\mathcal{E}_{\gamma} f_{L}(\xi)\right|>K^{-\epsilon}\left|\mathcal{E}_{\gamma} f(\xi)\right|\right.$ for some long horizontal strip $\left.L\right\}$,

$C:=\left\{\xi \in B_{R} \backslash B:\left|\mathcal{E}_{\gamma} f_{L}(\xi)\right|>K^{-\epsilon}\left|\mathcal{E}_{\gamma} f(\xi)\right|\right.$ for some long vertical strip $\left.L\right\}$ 
$D:=\left\{\xi \in B_{R} \backslash(B \cup C):\left|\mathcal{E}_{\gamma} f_{L}(\xi)\right|>K^{-\epsilon}\left|\mathcal{E}_{\gamma} f(\xi)\right|\right.$ for some short vertical strip $\left.L\right\}$.

By the definition of broad points, $B_{R}=A \cup B \cup C \cup D$. Notice also that if $|\gamma| K^{1 / 2} \geq 1$, then $C=D=\emptyset$ by construction.

If $\xi \in A$, then $\left|\mathcal{E}_{\gamma} f(\xi)\right|=B r_{K^{-\epsilon}} \mathcal{E}_{\gamma} f(\xi)$, so that the contribution of $A$ can be controlled using Theorem 2.1. Notice that

$$
\|f\|_{L^{2}(\Sigma)}^{12 / 13}\|f\|_{L^{\infty}(\Sigma)}^{1 / 13} \leq\|f\|_{L^{2}(\Sigma)}^{2 / q}\|f\|_{L^{\infty}(\Sigma)}^{1-2 / q},
$$

since $q>2.6>13 / 6$.

For the other parts, we induct on the size of $R$.

For $\xi \in B$ we estimate

$$
\left|\mathcal{E}_{\gamma} f(\xi)\right|<K^{\epsilon} \sup _{L}\left|\mathcal{E}_{\gamma} f_{L}(\xi)\right| \leq K^{\epsilon}\left(\sum_{L}\left|\mathcal{E}_{\gamma} f_{L}(\xi)\right|^{p}\right)^{1 / p},
$$

where here the supremum and sum are taken over all long horizontal strips $L$.

If $L=[0,1] \times\left[b, b+K^{-1 / 4}\right]$ is any of these long horizontal strips, we scale and translate $y=b+K^{-1 / 4} y^{\prime}$. Then

$$
K^{1 / 4} \phi_{\gamma}(x, y)=\left(x+\gamma K^{-1 / 4} b y^{\prime}\right) y^{\prime}+\frac{\gamma}{3 K^{1 / 2}} y^{\prime 3}+K^{1 / 4} b x+b^{2} \gamma y^{\prime}+\text { constant. }
$$

By applying the linear change of coordinates $x^{\prime}=x+\gamma K^{-1 / 4} b y^{\prime}$, we obtain

$$
K^{1 / 4} \phi_{\gamma}(x, y)=\phi_{\gamma / K^{1 / 2}}\left(x^{\prime}, y^{\prime}\right)+K^{1 / 4} b x^{\prime}+\text { constant. }
$$

Then

$$
\left|\mathcal{E}_{\gamma} f_{L}(\xi)\right|=K^{-1 / 4}\left|\mathcal{E}_{\gamma / K^{1 / 2}} f^{L}\left(\xi_{1}+b \xi_{3},\left(\xi_{2}-b \gamma \xi_{1}\right) K^{-1 / 4}, \xi_{3} K^{-1 / 4}\right)\right|,
$$

where we have defined $f^{L}$ by $f^{L}\left(x^{\prime}, y^{\prime}\right):=f_{L}(x, y)$, so that $\left\|f^{L}\right\|_{2}=K^{1 / 8}\left\|f_{L}\right\|_{2}$ and $\left\|f^{L}\right\|_{\infty} \leq\|f\|_{\infty}$. Note that we have $y^{\prime} \in[0,1]$ and $x^{\prime} \in[-1,2]$, since $\left|\gamma b K^{-1 / 4}\right| \leq 1$, and that the function $\mathcal{E}_{\gamma / K^{1 / 2}} f^{L}$ is supported in a box of dimensions $2 R \times \frac{2 R}{K^{1 / 4}} \times \frac{2 R}{K^{1 / 4}}$. What is crucial here is that, compared to $B_{R}$, this box is shorter by the factor $2 K^{-1 / 4} \leq 1 / 2$ in the $\xi_{3}$-direction, for $K$ sufficiently large.

A problem more of technical nature is that in $\xi_{1}$-direction it is still of the same size as $R$. However, as we shall show in Lemma 5.1, we can automatically pass from an estimate on a box $B_{R^{\prime}}$ to a corresponding estimate on the whole "plate" $P_{R^{\prime}}:=\mathbb{R}^{2} \times\left[0, R^{\prime}\right]$ containing $B_{R^{\prime}}$. Applying this in the present situation, with $R^{\prime}:=2 K^{-1 / 4} R \leq R / 2$, by our induction hypothesis we may then assume that the following estimate holds true:

$$
\left\|\mathcal{E}_{\gamma / K^{1 / 2}} f^{L}\right\|_{L^{3.25}\left(P_{R^{\prime}}\right)} \leq C_{\epsilon} R^{\prime \epsilon}\left\|f^{L}\right\|_{L^{2}(\Sigma)}^{2 / q}\left\|f^{L}\right\|_{L^{\infty}(\Sigma)}^{1-2 / q} .
$$

Thus, by (1) and (2), we see that

$$
\begin{aligned}
\left\|\mathcal{E}_{\gamma} f\right\|_{L^{p}(B)} & \leq K^{1 / 2 p-1 / 4+\epsilon}\left(\sum_{L}\left\|\mathcal{E}_{\gamma / K^{1 / 2}} f^{L}\right\|_{L^{p}\left(P_{2 R / K^{1 / 4}}\right)}^{p}\right)^{1 / p} \\
& \leq C C_{\epsilon} R^{\epsilon} K^{1 / 2 p-1 / 4 q^{\prime}+3 \epsilon / 4}\|f\|_{2}^{2 / q}\|f\|_{\infty}^{1-2 / q} \\
& \leq \frac{1}{10} C_{\epsilon} R^{\epsilon}\|f\|_{2}^{2 / q}\|f\|_{\infty}^{1-2 / q}
\end{aligned}
$$

since $p>2 q^{\prime}$. 
For $\xi \in C$, i.e., in the case of long vertical strips, we need to be a bit more careful. The natural change of coordinates is now $x=a+K^{-1 / 2} x^{\prime}$, if the long vertical strip $L$ is given by $L=\left[a, a+K^{-1 / 2}\right] \times[0,1]$. Then

$$
K^{1 / 2} \phi_{\gamma}(x, y)=x^{\prime} y+K^{1 / 2} \frac{\gamma}{3} y^{3}+a K^{1 / 2} y=\phi_{\gamma K^{1 / 2}}\left(x^{\prime}, y\right)+a K^{1 / 2} y,
$$

so to fit into our scheme, we need that $\left|\gamma K^{1 / 2}\right| \leq 1$. This is the reason why we consider this type of strips only when $\left|\gamma K^{1 / 2}\right| \leq 1$. Then we find that

$$
\left|\mathcal{E}_{\gamma} f_{L}(\xi)\right|=K^{-1 / 2}\left|\mathcal{E}_{\gamma K^{1 / 2}} f^{L}\left(\xi_{1} K^{-1 / 2}, \xi_{2}+a \xi_{3}, \xi_{3} K^{-1 / 2}\right)\right|,
$$

where $f^{L}$ is now defined by $f^{L}\left(x^{\prime}, y\right):=f_{L}(x, y)$, and can argue in a similar way as in the preceding case.

As for $D$, if $L=\left[a, a+K^{-1 / 2}\right] \times\left[b, b+K^{-1 / 4}\right]$ is any of the short vertical strips, then we scale and translate $x=a+K^{-1 / 2} x^{\prime}, y=b+K^{-1 / 4} y^{\prime}$. Then

$$
K^{3 / 4} \phi_{\gamma}(x, y)=\left(x^{\prime}+\gamma K^{1 / 4} b y^{\prime}\right)\left(y^{\prime}+K^{1 / 4} b\right)+\frac{\gamma}{3} y^{\prime 3}+K^{1 / 2} a y^{\prime}+\text { constant. }
$$

By applying the linear change of coordinates $x^{\prime \prime}=x^{\prime}+\gamma K^{1 / 4} b y^{\prime}, y^{\prime \prime}=y^{\prime}$ (note that, since $|\gamma| K^{1 / 2} \leq 1$, we have that $\left.|\gamma| b K^{1 / 4} \leq 1\right)$, we obtain

$$
K^{3 / 4} \phi_{\gamma}(x, y)=\phi_{\gamma}\left(x^{\prime \prime}, y^{\prime \prime}\right)+K^{1 / 4} b x^{\prime \prime}+K^{1 / 2} a y^{\prime \prime}+\text { constant. }
$$

Then, if $\xi \in E$,

$$
\left|\mathcal{E}_{\gamma} f_{L}(\xi)\right|=K^{-3 / 4}\left|\mathcal{E}_{\gamma} f^{L}\left(K^{-1 / 2}\left(\xi_{1}+b \xi_{3}\right), K^{-1 / 4}\left(\xi_{2}+a \xi_{3}-b \gamma \xi_{1}\right), K^{-3 / 4} \xi_{3}\right)\right|,
$$

where we have defined $f^{L}$ by $f^{L}\left(x^{\prime \prime}, y^{\prime \prime}\right):=f_{L}(x, y)$. From here on, we argue in a similar way as before.

\section{Reduction of Theorem 2.1 to a setup allowing for inductive arguments}

Following Section 3 in [14], we shall next devise a setup and formulate a more general statement in Theorem 3.2 which will become amenable to inductive arguments. As in that paper, we change and extend our previous notation slightly. We introduce a "multiplicity" $\mu \geq 1$, and choose accordingly caps $\tau$ which now are allowed to be squares of possibly larger side length $r_{\tau} \in\left[K^{-1}, \mu^{1 / 2} K^{-1}\right]$ than before. It can then happen that such a cap $\tau$ is no longer contained in $\Sigma$; in that case, we truncate it by replacing it with its intersection with $\Sigma$.

We assume that we are given a family of such caps $\tau$ covering $\Sigma=[0,1] \times[0,1]$ such that their centers are $K^{-1}$ - separated. Hence, at any point there will be at most $\mu$ of these caps which overlap at that point. Notice also that there are at most $K^{2}$ caps $\tau$ in the family. We also assume that we have a decomposition

$$
f=\sum_{\tau} f_{\tau},
$$

where $\operatorname{supp} f_{\tau} \subset \tau$. 
Given the family of caps, we define recursively a fixed family of ragged long horizontal strips $S_{\ell},\left(\ell=1,2, \ldots,\left[\mu^{-1 / 2} K^{1 / 4}\right]\right)$, of "widths" $\sim \mu^{1 / 2} K^{-1 / 4}$, in the following way:

$$
\begin{aligned}
\mathcal{F}_{1} & :=\left\{\tau: \tau^{0} \cap\left([0,1] \times\left[0, \mu^{1 / 2} K^{-1 / 4}\right]\right) \neq \emptyset\right\} \quad \text { and } S_{1}:=\bigcup_{\tau \in \mathcal{F}_{1}} \tau, \\
\mathcal{F}_{2} & :=\left\{\tau \notin \mathcal{F}_{1}: \tau^{0} \cap\left([0,1] \times\left[\mu^{1 / 2} K^{-1 / 4}, 2 \mu^{1 / 2} K^{-1 / 4}\right]\right) \neq \emptyset\right\} \quad \text { and } S_{2}:=\bigcup_{\tau \in \mathcal{F}_{2}} \tau, \\
& \vdots \\
\mathcal{F}_{\ell} & :=\left\{\tau \notin \cup_{j=1}^{\ell-1} \mathcal{F}_{j}: \tau^{0} \cap\left([0,1] \times\left[(\ell-1) \mu^{1 / 2} K^{-1 / 4}, \ell \mu^{1 / 2} K^{-1 / 4}\right]\right) \neq \emptyset\right\} \quad \text { and } \quad S_{\ell}:=\bigcup_{\tau \in \mathcal{F}_{\ell}} \tau,
\end{aligned}
$$

Here, $\tau^{0}$ denotes the open interior of $\tau$. Note that the families $\mathcal{F}_{\ell}$ are pairwise disjoint. Define $f_{S_{\ell}}:=\sum_{\tau \in \mathcal{F}_{\ell}} f_{\tau}$, so that $f=\sum_{\ell} f_{S_{\ell}}$.

When $|\gamma| K^{1 / 2} \leq 1$, we also define a family of pairwise in measure disjoint ragged long vertical strips of "widths" $\sim \mu^{1 / 2} K^{-1 / 2}$ in an analogous way, and a family of pairwise in measure disjoint ragged short vertical strips of dimensions $\sim \mu^{1 / 2} K^{-1 / 2} \times \mu^{1 / 2} K^{-1 / 4}$ given by all intersections of a long horizontal and a long vertical strip, and add them to our set of ragged strips by denoting them by $S_{\ell}, \ell=\left[\mu^{-1 / 2} K^{1 / 4}\right]+1, \ldots$, and put as before $f_{S_{\ell}}:=\sum_{\tau \in \mathcal{F}_{\ell}} f_{\tau}$.

Given a family of caps $\tau$ as above, and given the corresponding ragged strips $S_{\ell}$ and functions $f_{\tau}$ and $f_{S_{\ell}}$ as before, we say that a point $\xi \in \mathbb{R}^{3}$ is $\alpha$-broadfor $\mathcal{E}_{\gamma} f$ and the given family of caps, if

$$
\max _{S_{\ell}}\left|\mathcal{E}_{\gamma} f_{S_{\ell}}(\xi)\right| \leq \alpha\left|\mathcal{E}_{\gamma} f(\xi)\right|,
$$

where the maximum is taken over the set of all ragged strips $S_{\ell}$ as defined above (recall that this set depends on the size of $\left.|\gamma| K^{1 / 2}\right)$.

We also define $B r_{\alpha} \mathcal{E}_{\gamma} f(\xi):=\left|\mathcal{E}_{\gamma} f(\xi)\right|$ if $\xi$ is $\alpha$-broad, and zero otherwise.

Remark 3.1 Note that when $\mu=1$, then ragged strips are indeed strips in the sense of Definitions 2.1, and our present definition of broadness of points coincides in this case with the one given before.

The key result will be the following analogue to Theorem 3.1 in [14]:

Theorem 3.2 For any $0<\epsilon<10^{-10}$, there are constants $K=K(\epsilon)$ and $C_{\epsilon}$, independent of $\gamma \in[-1,1]$, such that for any family of caps $\tau$ with multiplicity at most $\mu$ covering $\Sigma$ as above and the associated family of ragged strips $S_{\ell}$ and associated functions $f_{\tau}$ and $f_{S_{\ell}}$ as defined above which decompose $f$, for any length $R \geq 1$, any $\alpha \geq K^{-\epsilon}$ and for any $\gamma \in[-1,1]$, the following holds true:

If for every $\omega \in \Sigma$, and every cap $\tau$ as above,

$$
\oint_{B\left(\omega, R^{-1 / 2}\right)}\left|f_{\tau}\right|^{2} \leq 1
$$

then,

$$
\int_{B_{R}}\left(B r_{\alpha} \mathcal{E}_{\gamma} f\right)^{3.25} \leq C_{\epsilon} R^{\epsilon}\left(\sum_{\tau} \int\left|f_{\tau}\right|^{2}\right)^{3 / 2+\epsilon} R^{\delta_{\text {trans }} \log \left(K^{\epsilon} \alpha \mu\right)},
$$


where $\delta_{\text {trans }}:=\epsilon^{6}$. Moreover $K(\epsilon) \rightarrow \infty$ as $\epsilon \rightarrow 0$.

Here, in $\mathbb{R}^{n}$, by $B(\omega, r)$ we denote the Euclidean ball of radius $r>0$ and center $\omega$, and by $\oint_{A} f:=\frac{1}{|A|} \int_{A} f$ we denote the mean value $f$ over the measurable set $A$ of volume $|A|>0$.

We can easily recover Theorem 2.1 by applying Theorem 3.2 with $\mu=1, \epsilon<10^{-10}$ and $\alpha=K^{-\epsilon}$, in the same way as Guth shows how Theorem 2.4 follows from Theorem 3.1 in [14]. Keep here Remark 3.1 in mind, and note that for these choices of $\mu, \epsilon$ and $\alpha$, we have $\delta_{\text {trans }} \log \left(2 K^{\epsilon} \alpha \mu\right) \leq 10 \delta_{\text {trans }} \leq \epsilon$.

\section{Proof of Theorem 3.2}

Recall that we had put $\delta_{\text {trans }}:=\epsilon^{6}$, so that, if we define $\delta_{\text {deg }}:=\epsilon^{4}$ and $\delta:=\epsilon^{2}$, then

$$
\delta_{\text {trans }} \ll \delta_{\text {deg }} \ll \delta \ll \epsilon<10^{-10} .
$$

We also set, for given $R \geq 1$,

$$
K=K(\epsilon):=e^{\epsilon^{-10}} \quad \text { and } \quad D=D(\epsilon):=R^{\delta_{\operatorname{deg}}}=R^{\epsilon^{4}} .
$$

Remark 4.1 a) It is enough to consider the case where $\alpha \mu \leq 10^{-5}$, because in the other case, the exponent $\delta_{\text {trans }} \log \left(K^{\epsilon} \alpha \mu\right)$ is very large and the estimate (5) trivially holds true. Henceforth, we shall therefore always assume that $\alpha \mu \leq 10^{-5}$.

b) It is then also enough to consider the case where $R \geq 1000 e^{e^{\epsilon^{-12}}}$.

To justify the last claim, notice first that our assumption (4) implies that $\left\|f_{\tau}\right\|_{2} \leq 1$. Since there are at most $K(\epsilon)^{2}$ caps $\tau$, we have $\sum_{\tau}\left\|f_{\tau}\right\|_{2} \leq K(\epsilon)^{2}$. Therefore, we trivially even obtain that when $R \leq 1000 e^{e^{\epsilon^{-12}}}$, then

$$
\begin{aligned}
\int_{B_{R}}\left|\mathcal{E}_{\gamma} f\right|^{3.25} & \leq R^{3}\|f\|_{1}^{3.25} \leq R^{3}\left(\sum_{\tau}\left\|f_{\tau}\right\|_{1}\right)^{3.25} \leq R^{3}\left(\sum_{\tau}\left\|f_{\tau}\right\|_{2}\right)^{3.25} \\
& \leq R^{3} K(\epsilon)^{2(1 / 4-2 \epsilon)}\left(\sum_{\tau}\left\|f_{\tau}\right\|_{2}\right)^{3+2 \epsilon} \\
& \leq R^{3} K(\epsilon)^{1 / 2-4 \epsilon} K(\epsilon)^{2(3 / 2+\epsilon)}\left(\sum_{\tau}\left\|f_{\tau}\right\|_{2}^{2}\right)^{3 / 2+\epsilon} \\
& \leq C_{1}(\epsilon)\left(\sum_{\tau}\left\|f_{\tau}\right\|_{2}^{2}\right)^{3 / 2+\epsilon},
\end{aligned}
$$

with $C_{1}(\epsilon):=\left(1000 e^{e^{\epsilon^{-12}}}\right)^{3} K(\epsilon)^{7 / 2-2 \epsilon}$, hence (5).

As usual, we will work with wave packet decompositions of the functions $f$ defined on $S_{\gamma}$. Following [14], we decompose $\Sigma$ into squares ("caps") $\theta$ of side length $R^{-1 / 2}$. By $\omega_{\theta}$ we shall denote the center of $\theta$, and by $v(\theta)$ the "outer" unit normal to $S_{\gamma}$ at the point $\left(\omega_{\theta}, \phi_{\gamma}\left(\omega_{\theta}\right)\right) \in S_{\gamma}$, which points into the direction of $\left(-\nabla \phi_{\gamma}\left(\omega_{\theta}\right),-1\right) . \mathbb{T}(\theta)$ will denote a set of $R^{1 / 2}$-separated tubes $T$ of radius $R^{1 / 2+\delta}$ and length $R$, which are all parallel to $v(\theta)$ and for which the corresponding thinner tubes of radius $R^{1 / 2}$ with the same axes cover $B_{R}$. We will write $v(T):=v(\theta)$ when $T \in \mathbb{T}(\theta)$.

Note that for each $\theta$, every point $\xi \in B_{R}$ lies in $O\left(R^{2 \delta}\right)$ tubes $T \in \mathbb{T}(\theta)$. We put $\mathbb{T}:=\bigcup_{\theta} \mathbb{T}(\theta)$. Arguing in the same way as in [14], Proposition 2.6, we arrive at the following approximate wave packet decomposition: 
Proposition 4.2 Assume that $R$ is sufficiently large (depending on $\delta$ ). Then, for any $\gamma \in$ $[-1,1]$, given $f \in L^{2}(\Sigma)$, we may associate to each tube $T \in \mathbb{T}$ a function $f_{T}$ such that the following hold true:

a) If $T \in \mathbb{T}(\theta)$, then $\operatorname{supp} f_{T} \subset 3 \theta$.

b) If $\xi \in B_{R} \backslash T$, then $\left|\mathcal{E}_{\gamma} f_{T}(\xi)\right| \leq R^{-1000}\|f\|_{2}$.

c) For any $x \in B_{R}$, we have $\left|\mathcal{E}_{\gamma} f(x)-\sum_{T \in \mathbb{T}} \mathcal{E}_{\gamma} f_{T}(x)\right| \leq R^{-1000}\|f\|_{2}$.

d) (Essential orthogonality) If $T_{1}, T_{2} \in \mathbb{T}(\theta)$ are disjoint, then

$\left|\int f_{T_{1}} \overline{f_{T_{2}}}\right| \leq R^{-1000} \int_{3 \theta}|f|^{2}$.

e) $\sum_{T \in \mathbb{T}(\theta)} \int_{\Sigma}\left|f_{T}\right|^{2} \leq C \int_{3 \theta}|f|^{2}$.

Remark 4.3 Note that since $|\gamma| \leq 1$, in this proposition we have bounds that are uniform in $\gamma$. Moreover, note that the same argument as in Remark $4.1 \mathrm{~b}$ ) shows that, in order to prove Theorem 3.2 it is enough to consider the case where $R$ is sufficiently large (depending on $\delta$, i.e., depending on $\epsilon$ ).

We next recall the version of the polynomial ham sandwich theorem with non-singular polynomials from [14]. If $P$ is a real polynomial on $\mathbb{R}^{n}$, we denote by $Z(P):=\left\{\xi \in \mathbb{R}^{n}\right.$ : $P(\xi)=0\}$ its null variety. $P$ is said to be non-singular if $\nabla P(\xi) \neq 0$ for every point $\xi \in Z(P)$.

Then, by Corollary 1.7 in [14] there is a non-zero polynomial $P$ of degree at most $D$ which is a product of non-singular polynomials such that the set $\mathbb{R}^{3} \backslash Z(P)$ is a disjoint union of $\sim D^{3}$ cells $O_{i}$ such that, for every $i$,

$$
\int_{O_{i} \cap B_{R}}\left(B r_{\alpha} \mathcal{E}_{\gamma} f\right)^{3.25} \sim D^{-3} \int_{B_{R}}\left(B r_{\alpha} \mathcal{E}_{\gamma} f\right)^{3.25} .
$$

We next define $W$ as the $R^{1 / 2+\delta}$ neighborhood of $Z(P)$ and put $O_{i}^{\prime}:=\left(O_{i} \cap B_{R}\right) \backslash W$.

Moreover, note that if we apply Proposition 4.2 to $f_{\tau}$ in place of $f$ (what we shall usually do), then by property (a) in Proposition 4.2, for every tube $T \in \mathbb{T}$ the function $f_{\tau, T}$ is supported in an $O\left(R^{-1 / 2}\right)$ neighborhood of $\tau$. Following Guth, we define

$\mathbb{T}_{i}:=\left\{T \in \mathbb{T}: T \cap O_{i}^{\prime} \neq \emptyset\right\}, \quad f_{\tau, i}:=\sum_{T \in \mathbb{T}_{i}} f_{\tau, T}, \quad f_{S_{\ell}, i}:=\sum_{\tau \in \mathcal{F}_{\ell}} f_{\tau, i} \quad$ and $f_{i}:=\sum_{\tau} f_{\tau, i}$.

Then we can use the following analogue to Lemma 3.2 in [14]:

Lemma 4.4 Each tube $T \in \mathbb{T}$ lies in at most $D+1$ of the sets $\mathbb{T}_{i}$.

We cover $B_{R}$ with $\sim R^{3 \delta}$ balls $B_{j}$ of radius $R^{1-\delta}$. Recall Definitions 3.3 and 3.4 from [14]:

Definition 4.1 a) We define $\mathbb{T}_{j, \text { tang }}$ as the set of all tubes $T \in \mathbb{T}$ that satisfy the following conditions:

$$
T \cap W \cap B_{j} \neq \emptyset,
$$

and if $\xi \in Z(P)$ is any nonsingular point (i.e., $\nabla P(\xi) \neq 0$ ) lying in $2 B_{j} \cap 10 T$, then

$$
\operatorname{angle}\left(v(T), T_{\xi} Z(P)\right) \leq R^{-1 / 2+2 \delta} .
$$

Here, $T_{\xi} Z(P)$ denotes the tangent space to $Z(P)$ at $\xi$, and we recall that $v(T)$ denotes the unit vector in direction of $T$. Accordingly, we define

$$
f_{\tau, j, \text { tang }}:=\sum_{T \in \mathbb{T}_{j, \text { tang }}} f_{\tau, T} \text { and } f_{j, \text { tang }}:=\sum_{\tau} f_{\tau, j, \text { tang }} .
$$


b) We define $\mathbb{T}_{j, \text { trans }}$ as the set of all tubes $T \in \mathbb{T}$ that satisfy the following conditions:

$$
T \cap W \cap B_{j} \neq \emptyset,
$$

and there exists a nonsingular point $\zeta \in Z(P)$ lying in $2 B_{j} \cap 10 T$, so that

$$
\operatorname{angle}\left(v(T), T_{\zeta} Z(P)\right)>R^{-1 / 2+2 \delta} .
$$

Accordingly, we define

$$
f_{\tau, j, \text { trans }}:=\sum_{T \in \mathbb{T}_{j, \text { trans }}} f_{\tau, T} \text { and } f_{j, \text { trans }}:=\sum_{\tau} f_{\tau, j, \text { trans }} .
$$

We also recall Lemmas 3.5 and 3.6 in [14]:

Lemma 4.5 Each tube $T \in \mathbb{T}$ belongs to at most $\operatorname{Poly}(D)=R^{O\left(\delta_{\text {deg }}\right)}$ different sets $\mathbb{T}_{j, \text { trans }}$.

Lemma 4.6 For each $j$, the number of different $\theta$ so that $\mathbb{T}_{j, \text { tang }} \cap \mathbb{T}(\theta) \neq \emptyset$ is at most $R^{1 / 2+O(\delta)}$.

Note that the previous lemma makes use of the fact that the Gaussian curvature does not vanish on the surface $S_{\gamma}$ so that the Gauß map is a diffeomorphism onto its image.

To motivate the next lemma, suppose we have a point $\xi$ contained in a cell $O_{i}^{\prime}$. Then it is not hard to see that in the wave packet decomposition of $\mathcal{E}_{\gamma} f(\xi)$ essentially only those tubes $T$ should matter which intersect the cell $O_{i}^{\prime}$, that is, $T \in \mathbb{T}_{i}$. It is thus natural to expect that we may replace $\mathcal{E}_{\gamma} f(\xi)$ by $\mathcal{E}_{\gamma} f_{i}(\xi)$ with only a small error. An analogous statement holds true even for the corresponding broad parts, as the following analogue to Lemma 3.7 in [14] shows:

Lemma 4.7 If $\xi \in O_{i}^{\prime}$. Then, given our assumptions on $R$ from Remarks 4.1, we have

$$
B r_{\alpha} \mathcal{E}_{\gamma} f(\xi) \leq B r_{2 \alpha} \mathcal{E}_{\gamma} f_{i}(\xi)+R^{-900} \sum_{\tau}\left\|f_{\tau}\right\|_{2}
$$

Proof Let $\xi \in O_{i}^{\prime}$. By Proposition $4.2 \mathrm{c}$ ), we have

$$
\mathcal{E}_{\gamma} f_{\tau}(\xi)=\sum_{T \in \mathbb{T}} \mathcal{E}_{\gamma} f_{\tau, T}(\xi)+O\left(R^{-1000}\left\|f_{\tau}\right\|_{2}\right) .
$$

If $\xi \in T$, then, $T \cap O_{i}^{\prime} \neq \emptyset$, i.e., $T \in \mathbb{T}_{i}$. If $\xi \notin T$, then Proposition 4.2 b) shows that $\left|\mathcal{E}_{\gamma} f_{\tau, T}(\xi)\right| \leq R^{-1000}\left\|f_{\tau}\right\|_{2}$. The contribution of these $T$ 's is thus negligible.

Using the short hand notation "neglig" for terms which are much smaller than $R^{-940} \sum_{\tau}\left\|f_{\tau}\right\|_{2}$ (and "neglig ${ }_{\tau}$ " for terms which are much smaller than $R^{-950}\left\|f_{\tau}\right\|_{2}$ ), we thus have

$$
\mathcal{E}_{\gamma} f_{\tau}(\xi)=\mathcal{E}_{\gamma} f_{\tau, i}(\xi)+\operatorname{neglig}_{\tau}
$$

and summing in $\tau$,

$$
\mathcal{E}_{\gamma} f(\xi)=\mathcal{E}_{\gamma} f_{i}(\xi)+\text { neglig. }
$$

We can assume that $\xi$ is $\alpha$-broad for $\mathcal{E}_{\gamma} f$ and that

$$
\left|\mathcal{E}_{\gamma} f(\xi)\right| \geq R^{-900} \sum_{\tau}\left\|f_{\tau}\right\|_{2} .
$$


Hence,

$$
\left|\mathcal{E}_{\gamma} f_{i}(\xi)\right| \geq\left|\mathcal{E}_{\gamma} f(\xi)\right|-\text { neglig } \geq \frac{1}{2} R^{-900} \sum_{\tau}\left\|f_{\tau}\right\|_{2} .
$$

Now assume that $S_{\ell}$ is any of the ragged strips used in the definition of $\alpha$-broadness. Then we have accordingly

$$
\mathcal{E}_{\gamma} f_{S_{\ell}}(\xi)=\sum_{\tau \in \mathcal{F}_{\ell}} \mathcal{E}_{\gamma} f_{\tau}(\xi)=\sum_{\tau \in \mathcal{F}_{\ell}} \mathcal{E}_{\gamma} f_{\tau, i}(\xi)+\text { neglig }=\mathcal{E}_{\gamma} f_{S_{\ell}, i}(\xi)+\text { neglig. }
$$

Since $\xi$ is $\alpha$-broad for $\mathcal{E}_{\gamma} f$, (11) shows that

$$
\left|\mathcal{E}_{\gamma} f_{S_{\ell}, i}(\xi)\right| \leq\left|\mathcal{E}_{\gamma} f_{S_{\ell}}(\xi)\right|+\text { neglig } \leq \alpha\left|\mathcal{E}_{\gamma} f(\xi)\right|+\text { neglig. }
$$

Notice also that by Remarks $4.1,10^{-5} \geq \alpha \gg K^{-\epsilon} \gg K^{-100} \gg R^{-1}$. In combination with (8), and (10), we then obtain that

$$
\left|\mathcal{E}_{\gamma} f_{S_{\ell}, i}(\xi)\right| \leq \alpha\left|\mathcal{E}_{\gamma} f_{i}(\xi)\right|+\text { neglig } \leq 2 \alpha\left|\mathcal{E}_{\gamma} f_{i}(\xi)\right|
$$

for every ragged $\operatorname{strip} S_{\ell}$. This estimate shows that $\xi$ is $2 \alpha$-broad for $\mathcal{E}_{\gamma} f_{i}$, and thus the claimed estimate in the lemma follows from (8) and the assumptions that we made subsequently.

Our definition of broadness of points was chosen differently from Guth's, since we shall also need a different notion of "non-adjacent" caps. This will be related to the validity of certain bilinear Fourier extension estimates which will be needed in the proof and which will be established later. In order to prepare those, let us review some notions and results concerning such bilinear estimates.

\subsection{Transversality for bilinear estimates}

We shall be brief here and refer for more details to the corresponding literature dealing with bilinear estimates, for instance [22,23,35], or [2].

Following in particular and more specifically our discussions in [8,9], we first recall that according to Theorem 1.1 in [22], given two open subsets $U_{1}, U_{2} \subset[0,1] \times[0,1]$, the proper type of transversality for bilinear estimates is achieved if the modulus of the following quantity

$$
\Gamma_{z}^{\gamma}\left(z_{1}, z_{2}, z_{1}^{\prime}, z_{2}^{\prime}\right):=\left\langle\left(H \phi_{\gamma}\right)^{-1}(z)\left(\nabla \phi_{\gamma}\left(z_{2}\right)-\nabla \phi_{\gamma}\left(z_{1}\right)\right), \nabla \phi_{\gamma}\left(z_{2}^{\prime}\right)-\nabla \phi_{\gamma}\left(z_{1}^{\prime}\right)\right\rangle
$$

is bounded from below for any $z_{i}=\left(x_{i}, y_{i}\right), z_{i}^{\prime}=\left(x_{i}^{\prime}, y_{i}^{\prime}\right) \in U_{i}, i=1,2$, and $z=(x, y) \in$ $U_{1} \cup U_{2}, H \phi_{\gamma}$ denoting the Hessian of $\phi$. If such an inequality holds, then we do have bilinear estimates with constants $C$ that depend only on lower bounds of (the modulus of) in (13), and on upper bounds for the derivatives of $\phi_{\gamma}$. Note that those upper bounds are independent of $\gamma \in[-1,1]$; we will be more precise about this later. If $U_{1}$ and $U_{2}$ are sufficiently small (with sizes depending on upper bounds of the first and second order derivatives of $\phi_{\gamma}$ and a lower bound for the determinant of $H \phi_{\gamma}$ ) this condition reduces to the estimate

$$
\left|\Gamma_{z}^{\gamma}\left(z_{1}, z_{2}\right)\right| \geq c>0
$$

for $z_{i}=\left(x_{i}, y_{i}\right) \in U_{i}, i=1,2, z=(x, y) \in U_{1} \cup U_{2}$, where

$$
\Gamma_{z}^{\gamma}\left(z_{1}, z_{2}\right):=\left\langle\left(H \phi_{\gamma}\right)^{-1}(z)\left(\nabla \phi_{\gamma}\left(z_{2}\right)-\nabla \phi_{\gamma}\left(z_{1}\right)\right), \nabla \phi_{\gamma}\left(z_{2}\right)-\nabla \phi_{\gamma}\left(z_{1}\right)\right\rangle .
$$


The bounds in the corresponding bilinear estimates will then depend on the lower bound $c$ in (14). In contrast to [8,9], where we had to devise quite specific "admissible pairs" of sets $U_{1}, U_{2}$ for our bilinear estimates, we shall here only have to consider caps $\tau_{1}, \tau_{2}$, and the required bilinear estimates will be a of somewhat different nature. Nevertheless, the geometric transversality conditions that we need here will be the same.

It is easy to check that we explicitly have

$$
\begin{aligned}
\Gamma_{z}^{\gamma}\left(z_{1}, z_{2}\right) & =2\left(y_{2}-y_{1}\right)\left[x_{2}-x_{1}+\gamma\left(y_{1}+y_{2}-y\right)\left(y_{2}-y_{1}\right)\right] \\
& =: 2\left(y_{2}-y_{1}\right) \mathbf{t}_{z}^{\gamma}\left(z_{1}, z_{2}\right) .
\end{aligned}
$$

Since $z=(x, y) \in U_{1} \cup U_{2}$, it will be particularly important to look at the expression (16) when $z=z_{1} \in U_{1}$, and $z=z_{2} \in U_{2}$. As above, if $U_{1}$ and $U_{2}$ are sufficiently small, we can actually reduce to this case. We then see that for our perturbed saddle, still the difference $y_{2}-y_{1}$ in the $y$-coordinates plays an important role as for the unperturbed saddle, but in place of the difference $x_{2}-x_{1}$ in the $x$-coordinates now the quantities

$$
\begin{aligned}
& \mathbf{t}_{z_{1}}^{\gamma}\left(z_{1}, z_{2}\right):=x_{2}-x_{1}+\gamma y_{2}\left(y_{2}-y_{1}\right) \\
& \mathbf{t}_{z_{2}}^{\gamma}\left(z_{1}, z_{2}\right):=x_{2}-x_{1}+\gamma y_{1}\left(y_{2}-y_{1}\right)
\end{aligned}
$$

become relevant. Observe also that

$$
\mathbf{t}_{z}^{\gamma}\left(z_{1}, z_{2}\right)=-\mathbf{t}_{z}^{\gamma}\left(z_{2}, z_{1}\right) .
$$

This definition of transversality motivates the following

Definition 4.2 a) We say that two caps $\tau_{1}, \tau_{2}$ are strongly separated if

$$
\min \left\{\left|y_{2}^{c}-y_{1}^{c}\right|, \max \left\{\left|\mathbf{t}_{z_{1}^{c}}^{\gamma}\left(z_{1}^{c}, z_{2}^{c}\right)\right|,\left|\mathbf{t}_{z_{2}^{c}}^{\gamma}\left(z_{1}^{c}, z_{2}^{c}\right)\right|\right\}\right\} \geq 10 \mu^{1 / 2} K^{-1},
$$

where $z_{1}^{c}=\left(x_{1}^{c}, y_{1}^{c}\right)$ denotes the center of $\tau_{1}$ and $z_{2}^{c}=\left(x_{2}^{c}, y_{2}^{c}\right)$ the center of $\tau_{2}$.

b) Following from here again [14], we define

$$
\operatorname{Bil}\left(\mathcal{E}_{\gamma} f_{j, \text { tang }}\right):=\sum_{\tau_{1}, \tau_{2} \text { strongly separated }}\left|\mathcal{E}_{\gamma} f_{\tau_{1}, j, \text { tang }}\right|^{1 / 2}\left|E_{\gamma} f_{\tau_{2}, j, \text { tang }}\right|^{1 / 2} .
$$

Remark 4.8 If the caps $\tau_{1}$ and $\tau_{2}$ are strongly separated, so that, say, $\left|y_{2}^{c}-y_{1}^{c}\right| \geq 10 \mu^{1 / 2} K^{-1}$ and $\left.\left|\mathbf{t}_{z_{2}^{c}}^{\gamma}\left(z_{1}^{c}, z_{2}^{c}\right)\right|\right\} \geq 10 \mu^{1 / 2} K^{-1}$, then by (16) we have

$$
\left|\Gamma_{z}^{\gamma}\left(z_{1}, z_{2}, z_{1}^{\prime}, z_{2}^{\prime}\right)\right| \geq 4 \mu K^{-2} \text { for all } z_{1}, z_{1}^{\prime} \in \tau_{1}, z, z_{2}, z_{2}^{\prime} \in \tau_{2} .
$$

Indeed, one computes that

$$
\begin{aligned}
\Gamma_{z}^{\gamma}\left(z_{1}, z_{2}, z_{1}^{\prime}, z_{2}^{\prime}\right)= & -2 \gamma y\left(y_{2}-y_{1}\right)\left(y_{2}^{\prime}-y_{1}^{\prime}\right) \\
& +\left(y_{2}^{\prime}-y_{1}^{\prime}\right)\left(x_{2}-x_{1}+\gamma\left(y_{2}^{2}-y_{1}^{2}\right)\right) \\
& +\left(y_{2}-y_{1}\right)\left(x_{2}^{\prime}-x_{1}^{\prime}+\gamma\left(\left(y_{2}^{\prime}\right)^{2}-\left(y_{1}^{\prime}\right)^{2}\right)\right) \\
= & \left(y_{2}^{\prime}-y_{1}^{\prime}\right) \mathbf{t}_{z}^{\gamma}\left(z_{1}, z_{2}\right)+\left(y_{2}-y_{1}\right) \mathbf{t}_{z}^{\gamma}\left(z_{1}^{\prime}, z_{2}^{\prime}\right),
\end{aligned}
$$

with $\mathbf{t}_{z}^{\gamma}\left(z_{1}, z_{2}\right)$ defined in (16).

Now, by (18), $\mathbf{t}_{z_{2}^{c}}^{\gamma}\left(z_{1}^{c}, z_{2}^{c}\right)=x_{2}^{c}-x_{1}^{c}+\gamma\left(y_{2}^{c}+y_{1}^{c}-y_{2}^{c}\right)\left(y_{2}^{c}-y_{1}^{c}\right)$, where $\left|\mathbf{t}_{z_{2}^{c}}^{\gamma}\left(z_{1}^{c}, z_{2}^{c}\right)\right| \geq$ $10 \mu^{1 / 2} K^{-1}$. Since the caps $\tau_{1}, \tau_{2}$ have side lengths $\leq \mu^{1 / 2} K^{-1}$, it is easily seen that $\left|\mathbf{t}_{z_{2}}^{\gamma}\left(z_{1}, z_{2}\right)-\mathbf{t}_{z_{2}^{c}}^{\gamma}\left(z_{1}^{c}, z_{2}^{c}\right)\right| \leq 8 \mu^{1 / 2} K^{-1}$, so that $\mathbf{t}_{z}^{\gamma}\left(z_{1}, z_{2}\right)$ and $\mathbf{t}_{z_{2}^{c}}^{\gamma}\left(z_{1}^{c}, z_{2}^{c}\right)$ have the same 
sign and $\left|\mathbf{t}_{z}^{\gamma}\left(z_{1}, z_{2}\right)\right| \geq 2 \mu^{1 / 2} K^{-1}$, and analogously we find that $\mathbf{t}_{z}^{\gamma}\left(z_{1}^{\prime}, z_{2}^{\prime}\right)$ and $\mathbf{t}_{z_{2}^{c}}^{\gamma}\left(z_{1}^{c}, z_{2}^{c}\right)$ have the same sign, and that $\left|\mathbf{t}_{z}^{\gamma}\left(z_{1}^{\prime}, z_{2}^{\prime}\right)\right| \geq 2 \mu^{1 / 2} K^{-1}$. In a similar way, we see that $\left(y_{2}^{\prime}-y_{1}^{\prime}\right)$ and $\left(y_{2}-y_{1}\right)$ have the same sign as $\left(y_{2}^{c}-y_{1}^{c}\right)$, and that $\min \left\{\left|y_{2}^{\prime}-y_{1}^{\prime}\right|,\left|y_{2}-y_{1}\right|\right\} \geq 2 \mu^{1 / 2} K^{-1}$, since $\left|y_{2}^{c}-y_{1}^{c}\right| \geq 10 \mu^{1 / 2} K^{-1}$. Therefore, $\left|\Gamma_{z}^{\gamma}\left(z_{1}, z_{2}, z_{1}^{\prime}, z_{2}^{\prime}\right)\right| \geq 2\left(2 \mu^{1 / 2} K^{-1}\right)^{2}$.

For any subset $I$ of the family of caps $\tau$, we define

$$
f_{I, j, \text { trans }}:=\sum_{\tau \in I} f_{\tau, j, \text { trans }} .
$$

The remaining part of this subsection will be devoted to the proof of the following crucial analogue to the key Lemma 3.8 in [14]:

Lemma 4.9 If $\xi \in B_{j} \cap W$ and $\alpha \mu \leq 10^{-5}$, then

$$
B r_{\alpha} \mathcal{E}_{\gamma} f(\xi) \leq 2\left(\sum_{I} B r_{60 \alpha} \mathcal{E}_{\gamma} f_{I, j, t \text { rans }}(\xi)+K^{100} \operatorname{Bil}\left(\mathcal{E}_{\gamma} f_{j, \text { tang }}\right)(\xi)+R^{-900} \sum_{\tau}\left\|f_{\tau}\right\|_{2}\right),
$$

where the first sum is over all possible subsets I of the given family of caps $\tau$.

Remark 4.10 The splitting into a "transversal" and "tangential" part here is as such not surprising. The crucial point is the presence of the bilinear term. In short, and oversimplified, a given family of caps $\tau$ will either contain two strongly separated caps, which gives rise to the bilinear term, or otherwise we will see by the Geometric Lemma 4.11 that the family cannot contain too many caps, and their contributions can be "bootstrapped" by means of Lemma 4.5. For the last point, broadness will be of utmost importance (compare (25)).

Proof Let $\xi \in B_{j} \cap W$. We may assume that $\xi$ is $\alpha$-broad for $\mathcal{E}_{\gamma} f$ and that $\left|\mathcal{E}_{\gamma} f(\xi)\right| \geq$ $R^{-900} \sum_{\tau}\|f\|_{2}$. Let

$$
I:=\left\{\tau:\left|\mathcal{E}_{\gamma} f_{\tau, j, \operatorname{tang}}(\xi)\right| \leq K^{-100}\left|\mathcal{E}_{\gamma} f(\xi)\right|\right\}
$$

We consider two possible cases:

Case 1: $I^{c}$ contains two strongly separated caps $\tau_{1}$ and $\tau_{2}$. Then trivially

$$
\left|\mathcal{E}_{\gamma} f(\xi)\right| \leq K^{100}\left|\mathcal{E}_{\gamma} f_{\tau_{1}, j, \text { tang }}(\xi)\right|^{1 / 2}\left|\mathcal{E}_{\gamma} f_{\tau_{2}, j, \text { tang }}(\xi)\right|^{1 / 2} \leq K^{100} \operatorname{Bil}\left(\mathcal{E}_{\gamma} f_{j, \text { tan }}\right)(\xi)
$$

hence (21).

Case 2: $I^{c}$ does not contain two strongly separated caps.

In this case, we shall make use of the following lemma whose proof will be postponed to Subsection 4.3. Recall the fixed family $\left\{S_{\ell}\right\}_{\ell}$ of of ragged strips that was associated to our given family of caps $\tau$ (covering $\Sigma$ ) in Section 3 .

Lemma 4.11 (The Geometric Lemma) Assume that $K \geq 20$, and let $I^{c}$ be any subfamily of the given family of caps which does not contain two strongly separated caps.

a) If $|\gamma| K^{1 / 2}>1$, then all of the caps of $I^{c}$ belong to the union of at most 40 of the families $\mathcal{F}_{m}$ associated to long horizontal ragged strips $S_{m}$ of width $\mu^{1 / 2} K^{-1 / 4}$.

b) If $|\gamma| K^{1 / 2} \leq 1$, then either all of the caps of $I^{c}$ belong to the union of at most 3 of families $\mathcal{F}_{m}$ associated to long horizontal ragged strips $S_{m}$ of width $\mu^{1 / 2} K^{-1 / 4}$, or all belong to the union of at most 40 of the families $\mathcal{F}_{m}$ associated to long vertical ragged strips $S_{m}$ of width $\mu^{1 / 2} K^{-1 / 2}$. 
Remark 4.12 Note that the two cases in a) and b) basically match with the corresponding distinction of cases in our definition of $\alpha$-broad points. For our subsequent argument this distinction will, however, not be relevant.

Using the Geometric Lemma we finish the proof of Lemma 4.9 as follows. We denote by $\left\{S_{m}\right\}_{m \in \mathcal{M}}$ the subset of at most 40 long ragged strips given by the Geometric Lemma. By

$$
J:=\bigcup_{m \in \mathcal{M}} \mathcal{F}_{m}
$$

we denote the corresponding subset of caps $\tau$. Then $I^{c} \subset J$, i.e., $J^{c} \subset I$. We write

$$
f=\sum_{m \in \mathcal{M}} \sum_{\tau \in \mathcal{F}_{m}} f_{\tau}+\sum_{\tau \in J^{c}} f_{\tau} .
$$

Hence,

$$
\left|\mathcal{E}_{\gamma} f(\xi)\right| \leq \sum_{m \in \mathcal{M}}\left|\mathcal{E}_{\gamma} f_{S_{m}}(\xi)\right|+\left|\sum_{\tau \in J^{c}} \mathcal{E}_{\gamma} f_{\tau}(\xi)\right| .
$$

Since $\xi$ is $\alpha$-broad,

$$
\sum_{m \in \mathcal{M}}\left|\mathcal{E}_{\gamma} f_{S_{m}}(\xi)\right| \leq \sum_{m \in \mathcal{M}} \alpha\left|\mathcal{E}_{\gamma} f(\xi)\right| \leq 40 \alpha\left|\mathcal{E}_{\gamma} f(\xi)\right| \leq \frac{1}{10}\left|\mathcal{E}_{\gamma} f(\xi)\right|,
$$

where the last inequality holds because we are assuming that $\alpha \leq 10^{-5}$ (compare Remark 4.1 a)). Thus,

$$
\left|\mathcal{E}_{\gamma} f(\xi)\right| \leq \frac{1}{10}\left|\mathcal{E}_{\gamma} f(\xi)\right|+\left|\sum_{\tau \in J^{c}} \mathcal{E}_{\gamma} f_{\tau}(\xi)\right|
$$

and therefore

$$
\left|\mathcal{E}_{\gamma} f(\xi)\right| \leq \frac{10}{9}\left|\sum_{\tau \in J^{c}} \mathcal{E}_{\gamma} f_{\tau}(\xi)\right| .
$$

Since $\xi \in B_{j} \cap W$, by Proposition 4.2,

$$
\mathcal{E}_{\gamma} f_{\tau}(\xi)=\mathcal{E}_{\gamma} f_{\tau, j, \text { trans }}(\xi)+\mathcal{E}_{\gamma} f_{\tau, j, \text { tang }}(\xi)+O\left(R^{-1000}\right)\left\|f_{\tau}\right\|_{2} .
$$

Moreover, since $J^{c} \subset I$, and since there are at most $K^{2}$ caps $\tau$,

$$
\sum_{\tau \in J^{c}}\left|\mathcal{E}_{\gamma} f_{\tau, j, \text { tang }}(\xi)\right| \leq \sum_{\tau \in I}\left|\mathcal{E}_{\gamma} f_{\tau, j, \text { tang }}(\xi)\right| \leq K^{-100} \sum_{\tau \in I}\left|\mathcal{E}_{\gamma} f(\xi)\right| \leq K^{-98}\left|\mathcal{E}_{\gamma} f(\xi)\right|,
$$

where the second inequality is a consequence of the definition of $I$. Thus,

$$
\begin{aligned}
\frac{9}{10}\left|\mathcal{E}_{\gamma} f(\xi)\right| & \leq\left|\sum_{\tau \in J^{c}} \mathcal{E}_{\gamma} f_{\tau, j, \text { trans }}(\xi)\right|+K^{-98}\left|\mathcal{E}_{\gamma} f(\xi)\right|+\sum_{\tau} R^{-1000}\left\|f_{\tau}\right\|_{2} \\
& =\left|\mathcal{E}_{\gamma} f_{J^{c}, j, \text { trans }}(\xi)\right|+K^{-98}\left|\mathcal{E}_{\gamma} f(\xi)\right|+\sum_{\tau} R^{-1000}\left\|f_{\tau}\right\|_{2},
\end{aligned}
$$

and hence, since $\left|\mathcal{E}_{\gamma} f(\xi)\right| \geq R^{-900} \sum\left\|f_{\tau}\right\|_{2}$,

$$
\left|\mathcal{E}_{\gamma} f(\xi)\right| \leq \frac{11}{9}\left|\mathcal{E}_{\gamma} f_{J^{c}, j, t \operatorname{trans}}(\xi)\right| .
$$


Fig. 1 Intersection of long horizontal and long vertical strips (color figure online)

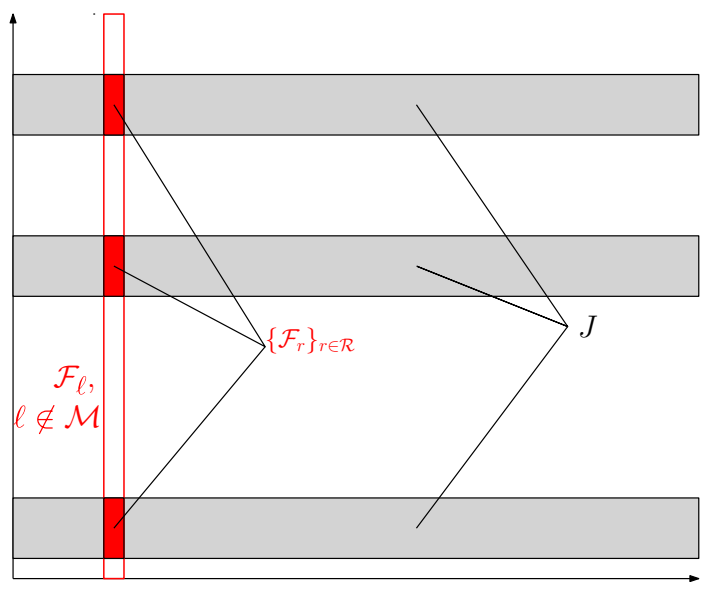

It will then finally suffice to show that $\xi$ is $60 \alpha$-broad for $\mathcal{E}_{\gamma} g$, where $g:=f_{J^{c}, j, \text { trans }}$. To this end let us set $g_{\tau}:=f_{\tau, j, \text { trans }}$, if $\tau \in J^{c}$, and zero otherwise, so that

$$
g=\sum g_{\tau}
$$

Observe first that by (26)

$$
\left|\mathcal{E}_{\gamma} f_{\tau, j, \operatorname{trans}}(\xi)\right| \leq\left|\mathcal{E}_{\gamma} f_{\tau}(\xi)\right|+\left|\mathcal{E}_{\gamma} f_{\tau, j, \text { tang }}(\xi)\right|+\text { neglig },
$$

so that if $\tau \in J^{c} \subset I$, then by the definition of $I$,

$$
\left|\mathcal{E}_{\gamma} f_{\tau, j, \text { trans }}(\xi)\right| \leq\left|\mathcal{E}_{\gamma} f_{\tau}(\xi)\right|+K^{-100}\left|\mathcal{E}_{\gamma} f(\xi)\right|+\text { neglig. }
$$

We have to show that

$$
\left|\mathcal{E}_{\gamma} g_{S_{\ell}}(\xi)\right| \leq 60 \alpha\left|\mathcal{E}_{\gamma} g(\xi)\right|
$$

for all ragged strips $S_{\ell}$. But, $g_{S_{\ell}}=\sum_{\tau \in \mathcal{F}_{\ell} \cap J^{c}} f_{\tau, j, t \text { trans }}$, and therefore the following two cases can arise:

(i) If $\ell \in \mathcal{M}$, then $\mathcal{F}_{\ell} \cap J^{c}=\emptyset$.

(ii) If $\ell \notin \mathcal{M}$, then by our construction of the set $J$ there is a collection $\left\{\mathcal{F}_{r}\right\}_{r \in \mathcal{R}}$ of at most 40 families (possibly empty) associated to short vertical ragged strips so that $\mathcal{F}_{\ell} \cap J=\cup_{\mathcal{R}} \mathcal{F}_{r}$ (cf. Fig. 1).

In case (i), (30) is trivial. In case (ii), observe first that by summing (26) over all $\tau \in \mathcal{F}_{\ell} \cap J^{c}$ we obtain

$$
\left|\mathcal{E}_{\gamma} g_{S_{\ell}}(\xi)\right| \leq\left|\sum_{\tau \in \mathcal{F}_{\ell} \cap J^{c}} \mathcal{E}_{\gamma} f_{\tau}(\xi)\right|+\sum_{\tau \in \mathcal{F}_{\ell} \cap J^{c}}\left|\mathcal{E}_{\gamma} f_{\tau, j, t a n g}(\xi)\right|+\text { neglig. }
$$

By (27), the second term can again be estimated by

$$
\sum_{\tau \in \mathcal{F}_{\ell} \cap J^{c}}\left|\mathcal{E}_{\gamma} f_{\tau, j, \text { tang }}(\xi)\right| \leq K^{-98}\left|\mathcal{E}_{\gamma} f(\xi)\right| .
$$

We write

$$
\sum_{\tau \in \mathcal{F}_{\ell} \cap J^{c}} \mathcal{E}_{\gamma} f_{\tau}(\xi)=\mathcal{E}_{\gamma} f_{S_{\ell}}(\xi)-\sum_{r \in \mathcal{R}} \mathcal{E}_{\gamma} f_{S_{r}}(\xi)
$$


Since $\xi$ is $\alpha$-broad for $\mathcal{E}_{\gamma} f$, both terms are estimated using again broadness:

$$
\left|\sum_{\tau \in \mathcal{F}_{\ell} \cap J^{c}} \mathcal{E}_{\gamma} f_{\tau}(\xi)\right| \leq 41 \alpha\left|\mathcal{E}_{\gamma} f(\xi)\right| .
$$

Since $\alpha \geq K^{-\epsilon} \gg 10 K^{-98}$, in combination with (28) we conclude that

$$
\begin{aligned}
\left|\mathcal{E}_{\gamma} g_{S_{\ell}}(\xi)\right| & \leq 41 \alpha\left|\mathcal{E}_{\gamma} f(\xi)\right|+K^{-98}\left|\mathcal{E}_{\gamma} f(\xi)\right|+\text { neglig } \leq(41+1 / 2) \alpha\left|\mathcal{E}_{\gamma} f(\xi)\right| \\
& \leq 60 \alpha\left|\mathcal{E}_{\gamma} f_{J^{c}, j, \text { trans }}(\xi)\right|=60 \alpha\left|\mathcal{E}_{\gamma} g(\xi)\right| .
\end{aligned}
$$

This completes the proof of Lemma 4.9.

The contribution by the bilinear term in (21) will be controlled by means of the following analogue to Proposition 3.9 in [14]:

Proposition 4.13 We have

$$
\int_{B_{j} \cap W} \operatorname{Bil}\left(\mathcal{E}_{\gamma} f_{j, \text { tang }}\right)^{3.25} \leq C_{\epsilon} R^{O(\delta)+\epsilon / 2}\left(\sum_{\tau} \int\left|f_{\tau}\right|^{2}\right)^{3 / 2+\epsilon} .
$$

With Proposition 4.13 at hand, the rest of the proof of Theorem 3.2, which we shall detail in the next subsection, will be a literal copy of the arguments in pages 396-398 of [14].

The proof of this proposition can easily be reduced to the following analogue to Lemma 3.10 in [14]. We shall give some details below. It is in this lemma where we shall need the full thrust of the strong separation condition between caps $\tau_{1}$ and $\tau_{2}$. Suppose we have covered $B_{j} \cap W$ with a minimal number of cubes $Q$ of side length $R^{1 / 2}$, and denote by $\mathbb{T}_{j, \text { tang, } Q}$ the set of all tubes $T$ in $\mathbb{T}_{j, \text { tang }}$ such that $10 T$ intersects $Q$.

Lemma 4.14 Fix $j$, i.e., a ball $B_{j}$. If $\tau_{1}, \tau_{2}$ are strongly separated caps, then for any of the cubes $Q$ we have

$$
\begin{aligned}
& \int_{Q}\left|\mathcal{E}_{\gamma} f_{\tau_{1}, j, \text { tang }}\right|^{2}\left|\mathcal{E}_{\gamma} f_{\tau_{2}, j, \text { tang }}\right|^{2} \\
& \quad \leq R^{O(\delta)} R^{-1 / 2}\left(\sum_{T_{1} \in \mathbb{T}_{j, \text { tang }, Q}}\left\|f_{\tau_{1}, T_{1}}\right\|_{2}^{2}\right)\left(\sum_{T_{2} \in \mathbb{T}_{j, \text { tang, },}}\left\|f_{\tau_{2}, T_{2}}\right\|_{2}^{2}\right)+\text { neglig. }
\end{aligned}
$$

Indeed, the main ingredient in Guth's argument that needs to be checked here is the following geometric property (compare p. 402 in [14]):

Lemma 4.15 If $\tau_{1}$ and $\tau_{2}$ are two strongly separated caps, then, for any two points $z_{1}=$ $\left(x_{1}, y_{1}\right) \in \tau_{1}$ and $z_{2}=\left(x_{2}, y_{2}\right) \in \tau_{2}$ the angle between the normals to $S_{\gamma}$ at the corresponding points on $S_{\gamma}$ is $\gtrsim K^{-1}$.

Proof By $N_{\gamma}(x, y)$ we denote the following normal to our surface $S_{\gamma}$ at $\left(x, y, \phi_{\gamma}(x, y)\right) \in$ $S_{\gamma}:$

$$
N_{\gamma}(x, y):=\left(\begin{array}{c}
{ }^{t} \nabla \phi_{\gamma}(x, y) \\
-1
\end{array}\right)
$$

Note that these normal vectors are of size $\left|N_{\gamma}(x, y)\right| \sim 1$. Since $\nabla \phi_{\gamma}(x, y)=\left(y, x+\gamma y^{2}\right)$, we see that

$$
\left|\nabla \phi_{\gamma}\left(x_{2}, y_{2}\right)-\nabla \phi_{\gamma}\left(x_{1}, y_{1}\right)\right| \geq\left|y_{2}-y_{1}\right| \gtrsim 10 K^{-1}
$$

since $\tau_{1}$ and $\tau_{2}$ are strongly separated. This implies the claim about the angle. 
With this at hand, we can follow Guth to deduce from Lemma 4.14 the following $L^{4}$ estimate

$$
\left\|\operatorname{Bil}\left(\mathcal{E}_{\gamma} f_{j, \text { tang }}\right)\right\|_{L^{4}\left(B_{j} \cap W\right)} \leq R^{O(\delta)} R^{-1 / 8}\left(\sum_{\tau}\left\|f_{\tau, j, \tan }\right\|_{2}^{2}\right)^{1 / 2}+\text { neglig, }
$$

which corresponds to inequality (43) in [14]. Indeed, we can use the standard estimate

$$
\left\|\mathcal{E}_{\gamma} f\right\|_{L^{2}\left(B_{R}\right)} \lesssim R^{1 / 2}\|f\|_{2}
$$

to deduce that

$$
\left\|\operatorname{Bil}\left(\mathcal{E}_{\gamma} f_{j, \text { tang }}\right)\right\|_{L^{2}\left(B_{j} \cap W\right)} \leq R^{1 / 2}\left(\sum_{\tau}\left\|f_{\tau, j, \text { tan }}\right\|_{2}^{2}\right)^{1 / 2} .
$$

From this and (33), by Hölder's inequality we get for $2 \leq p \leq 4$,

$$
\int_{B_{j} \cap W} \operatorname{Bil}\left(\mathcal{E}_{\gamma} f_{j, \text { tang }}\right)^{p} \lesssim R^{O(\delta)} R^{\frac{5}{2}-\frac{3}{4} p}\left(\sum_{\tau}\left\|f_{\tau, j, \text { tang }}\right\|_{2}^{2}\right)^{p / 2}+\text { neglig. }
$$

Lemma 4.6 tells us that $\mathbb{T}_{j, \text { tang }}$ contains tubes in only $R^{O(\delta)} R^{1 / 2}$ directions. Hence, each function $f_{\tau, j, \text { tang }}$ is supported on at most $R^{O(\delta)} R^{1 / 2}$ caps $\theta$. By Proposition 4.2,

$$
\oint_{\theta}\left|f_{\tau, j, t a n g}\right|^{2} \lesssim \oint_{10 \theta}\left|f_{\tau}\right|^{2} \lesssim 1
$$

Adding the contribution of $R^{O(\delta)} R^{1 / 2}$ caps $\theta$, we get $\int\left|f_{\tau, j, t a n g}\right|^{2} \leq C R^{O(\delta)} R^{-1 / 2}$. Since there are $K^{2} \ll R^{O(\delta)}$ caps $\tau$, this implies that $\sum_{\tau}\left\|f_{\tau, j, \text { tang }}\right\|_{2}^{2} \leq C R^{O(\delta)} R^{-1 / 2}$. Hence, we get, for $p>3, \epsilon<2(p-3)$,

$$
\int_{B_{j} \cap W} \operatorname{Bil}\left(\mathcal{E}_{\gamma} f_{j, \text { tang }}\right)^{p} \lesssim R^{O(\delta)} R^{\frac{5}{2}-\frac{3}{4} p-\frac{1}{2}\left(\frac{p}{2}-\frac{3}{2}-\epsilon\right)}\left(\sum_{\tau}\left\|f_{\tau, j, \text { tang }}\right\|_{2}^{2}\right)^{3 / 2+\epsilon} .
$$

This finishes the proof of Proposition 4.13, for $p=3.25=\frac{13}{4}$.

Proof of Lemma 4.14. Let $\tau_{1}$ and $\tau_{2}$ be two strongly separated caps, and assume without loss of generality that $\min \left\{\left|y_{2}^{c}-y_{1}^{c}\right|,\left|\mathbf{t}_{z_{2}^{c}}^{\gamma}\left(z_{1}^{c}, z_{2}^{c}\right)\right|\right\} \geq 10 \mu^{1 / 2} K^{-1}$, where $z_{1}^{c}=\left(x_{1}^{c}, y_{1}^{c}\right)$ denotes the center of $\tau_{1}$ and $z_{2}^{c}=\left(x_{2}^{c}, y_{2}^{c}\right)$ the center of $\tau_{2}$.

Following in a first step a standard argument as in [14] based on Plancherel's theorem, and making use of Proposition 4.2 we see that

$$
\begin{aligned}
& \int_{Q}\left|\mathcal{E}_{\gamma} f_{\tau_{1}, j, \text { tang }}\right|^{2}\left|\mathcal{E}_{\gamma} f_{\tau_{2}, j, \text { tang }}\right|^{2} \\
& \leq \sum_{T_{1}, T_{1}^{\prime}, T_{2}, T_{2}^{\prime} \in \mathbb{T}_{j, \text { tang }, Q}} \int \mathcal{E}_{\gamma} f_{\tau_{1}, T_{1}} \mathcal{E}_{\gamma} f_{\tau_{2}, T_{2}} \overline{\mathcal{E}_{\gamma} f_{\tau_{1}^{\prime}, T_{1}^{\prime}} \mathcal{E}_{\gamma} f_{\tau_{2}^{\prime}, T_{2}^{\prime}}}+\text { neglig } \\
& =\sum_{T_{1}, T_{1}^{\prime}, T_{2}, T_{2}^{\prime} \in \mathbb{T}_{j, \text { tang }, Q}}\left(f_{\tau_{1}, T_{1}} d \sigma_{\gamma} * f_{\tau_{2}, T_{2}} d \sigma_{\gamma}\right) \overline{\left(f_{\tau_{1}^{\prime}, T_{1}^{\prime}} d \sigma_{\gamma} * f_{\tau_{2}^{\prime}, T_{2}^{\prime}} d \sigma_{\gamma}\right)}+\text { neglig. }
\end{aligned}
$$

Here $\sigma_{\gamma}$ denotes the surface carried measure on $S_{\gamma}$ chosen so that $\mathcal{E}_{\gamma} f=\widehat{\tilde{f} d \sigma_{\gamma}}$, if we set $\tilde{f}\left(z, \phi_{\gamma}(z)\right):=f(z)$.

For each tube $T$, we denote by $\theta(T)$ the cap $\theta$ so that $T \in \mathbb{T}(\theta)$, and let $\omega(T)$ be the center of $\theta(T)$. By $\tilde{\omega}(T):=\left(\omega(T), \phi_{\gamma}(\omega(T))\right.$ we denote the corresponding point on $S_{\gamma}$. 
A given term in the first sum is not negligible only if there are four points $z_{1}, z_{1}^{\prime} \in \tau_{1}$, $z_{2}, z_{2}^{\prime} \in \tau_{2}$ that satisfy

$$
\left(z_{1}, \phi_{\gamma}\left(z_{1}\right)\right)+\left(z_{2}, \phi_{\gamma}\left(z_{2}\right)\right)=\left(z_{1}^{\prime}, \phi_{\gamma}\left(z_{1}^{\prime}\right)\right)+\left(z_{2}^{\prime}, \phi_{\gamma}\left(z_{2}^{\prime}\right)\right)
$$

and

$$
\left(z_{i}, \phi_{\gamma}\left(z_{i}\right)\right)=\tilde{\omega}\left(T_{i}\right)+O\left(R^{-1 / 2+\delta}\right), \quad\left(z_{i}^{\prime}, \phi_{\gamma}\left(z_{i}^{\prime}\right)\right)=\tilde{\omega}\left(T_{i}^{\prime}\right)+O\left(R^{-1 / 2+\delta}\right), \quad i=1,2 .
$$

Let us denote by $\mathbf{S}_{i}$ the piece of the surface $S_{\gamma}$ corresponding to $\tau_{i}, i=1,2$ (which are "genuine" caps). Since the caps $\tau_{1}$ and $\tau_{2}$ are strongly separated, by Lemma 4.15 these two subsurfaces are transversal, so that we can locally define the intersection curve

$$
\Pi_{z_{1}, z_{2}^{\prime}}:=\left[\mathbf{S}_{1}+\left(z_{2}^{\prime}, \phi\left(z_{2}^{\prime}\right)\right)\right] \cap\left[\mathbf{S}_{2}+\left(z_{1}, \phi\left(z_{1}\right)\right)\right] .
$$

Note that by (35)

$$
\left(z_{1}, \phi_{\gamma}\left(z_{1}\right)\right)+\left(z_{2}, \phi_{\gamma}\left(z_{2}\right)\right)=\left(z_{1}^{\prime}, \phi_{\gamma}\left(z_{1}^{\prime}\right)\right)+\left(z_{2}^{\prime}, \phi_{\gamma}\left(z_{2}^{\prime}\right)\right) \in \Pi_{z_{1}, z_{2}^{\prime}} .
$$

Set $\psi(z):=\phi_{\gamma}\left(z-z_{1}\right)+\phi_{\gamma}\left(z_{1}\right)-\phi_{\gamma}\left(z-z_{2}^{\prime}\right)-\phi_{\gamma}\left(z_{2}^{\prime}\right)$. Then, the orthogonal projection of the curve $\Pi_{z_{1}, z_{2}^{\prime}}$ on the $z$ - plane is the curve given by $\{z: \psi(z)=0\}$ (just consider $z:=z_{1}+z_{2}=z_{1}^{\prime}+z_{2}^{\prime}$ for $z$ when (35) is satisfied).

We introduce a parametrization by arc length $z(t), t \in J$, of this curve, where $t$ is from an open interval $J$. Notice that this curve $z(t)$ depends on the choices of the points $z_{1}$ and $z_{2}^{\prime}$. By

$$
z_{1}^{\prime}(t):=z(t)-z_{2}^{\prime} \quad \text { and } \quad z_{2}(t):=z(t)-z_{1}
$$

we denote the corresponding curves on $\mathbf{S}_{1}$ and $\mathbf{S}_{2}$, respectively. We may assume that $0 \in J$ and $z_{1}^{\prime}(0)=z_{1}^{\prime}, z_{2}(0)=z_{2}$. Then, for $z_{1}, z_{2}^{\prime}$ fixed, the pairs $\left(z_{1}^{\prime}(t), z_{2}(t)\right), t \in J$, locally provide all solutions $\left(z_{1}^{\prime}, z_{2}\right)$ to (35).

Note that $\psi(z(t)) \equiv 0$ implies that

$$
\left\langle\nabla \phi_{\gamma}\left(z_{2}(t)\right)-\nabla \phi_{\gamma}\left(z_{1}^{\prime}(t)\right), \frac{d z}{d t}(t)\right\rangle=0 \text { for every } t \in J .
$$

Note also that $\left(z_{1}^{\prime}, z_{2}\right):=\left(z_{1}, z_{2}^{\prime}\right)$ is a solution of (35), so that we may assume that there is some $t_{2} \in J$ such that $z_{2}^{\prime}=z_{2}\left(t_{2}\right)$. Recall also that $z_{2}(0)=z_{2}$.

Recall the normal $N_{\gamma}(x, y)$ to the surface $S_{\gamma}$ at the point $\left(x, y, \phi_{\gamma}(x, y)\right) \in S_{\gamma}$ from (31), and note that the angle between the tube $T_{i}$ and $N_{\gamma}\left(z_{i}\right), i=1,2$, is bounded by $R^{-1 / 2}$.

Since $T_{1}, T_{2}, T_{1}^{\prime}, T_{2}^{\prime}$ lie in $\mathbb{T}_{j, \text { tang, } Q}$, we then obviously have

$$
\begin{aligned}
R^{-1 / 2+2 \delta} & \geq\left|\operatorname{det}\left(N_{\gamma}\left(z_{1}\right), N_{\gamma}\left(z_{2}\right), N_{\gamma}\left(z_{2}^{\prime}\right)\right)\right|=\left|\operatorname{det}\left(N_{\gamma}\left(z_{1}\right), N_{\gamma}\left(z_{2}\right), N_{\gamma}\left(z_{2}^{\prime}\right)-N_{\gamma}\left(z_{2}\right)\right)\right| \\
& =\left|\int_{0}^{t_{2}} \operatorname{det}\left(N\left(z_{1}\right), N\left(z_{2}\right), \frac{d N_{\gamma}\left(z_{2}(t)\right)}{d t}\right) d t\right| .
\end{aligned}
$$

For a given $t$,

$$
\begin{aligned}
& \operatorname{det}\left(N_{\gamma}\left(z_{1}\right), N_{\gamma}\left(z_{2}\right), \frac{d N_{\gamma}\left(z_{2}(t)\right)}{d t}\right)=\operatorname{det}\left(\begin{array}{cc}
{ }^{t} \nabla \phi_{\gamma}\left(z_{1}\right) & { }^{t} \nabla \phi_{\gamma}\left(z_{2}\right) H \phi_{\gamma}\left(z_{2}(t)\right) \cdot{ }^{t}\left(\frac{d z}{d t}(t)\right) \\
-1 & -1
\end{array}\right) \\
& =\operatorname{det}\left({ }^{t} \nabla \phi_{\gamma}\left(z_{1}\right)-{ }^{t} \nabla \phi_{\gamma}\left(z_{2}\right), H \phi_{\gamma}\left(z_{2}(t)\right) \cdot{ }^{t}\left(\frac{d z}{d t}(t)\right)\right) \\
& \left.=\operatorname{det} H \phi_{\gamma}\left(z_{2}(t)\right) \operatorname{det}\left(H \phi_{\gamma}\left(z_{2}(t)\right)\right)^{-1} \cdot\left({ }^{t} \nabla \phi_{\gamma}\left(z_{1}\right)-{ }^{t} \nabla \phi_{\gamma}\left(z_{2}\right)\right),{ }^{t}\left(\frac{d z}{d t}(t)\right)\right) .
\end{aligned}
$$


Since $\left.\mid \frac{d z}{d t}(t)\right) \mid=1$ and $\operatorname{det} H \phi_{\gamma}(z(t))=1$, in combination with (37) we thus see that

$$
\begin{aligned}
& \left|\operatorname{det}\left(N_{\gamma}\left(z_{1}\right), N_{\gamma}\left(z_{2}\right), \frac{d N_{\gamma}\left(z_{2}(t)\right)}{d t}\right)\right| \\
& =\frac{\left|\left\langle H \phi_{\gamma}\left(z_{2}(t)\right)^{-1}\left({ }^{t} \nabla \phi\left(z_{1}\right)-{ }^{t} \nabla \phi\left(z_{2}\right)\right), \nabla \phi_{\gamma}\left(z_{2}(t)\right)-\nabla \phi_{\gamma}\left(z_{1}^{\prime}(t)\right)\right\rangle\right|}{\left|\nabla \phi_{\gamma}\left(z_{2}(t)\right)-\nabla \phi_{\gamma}\left(z_{1}^{\prime}(t)\right)\right|} \\
& =\frac{\left|\Gamma_{z_{2}(t)}^{\gamma}\left(z_{1}, z_{2}, z_{1}^{\prime}(t), z_{2}(t)\right)\right|}{\left|\nabla \phi_{\gamma}\left(z_{2}(t)\right)-\nabla \phi_{\gamma}\left(z_{1}^{\prime}(t)\right)\right|} .
\end{aligned}
$$

Note that here $\left|\nabla \phi_{\gamma}\left(z_{2}(t)\right)-\nabla \phi_{\gamma}\left(z_{1}^{\prime}(t)\right)\right| \leq 4$. Moreover, by our assumptions and Remark 4.8, we have $\left|\Gamma_{z_{2}(t)}^{\gamma}\left(z_{1}, z_{2}, z_{1}^{\prime}(t), z_{2}(t)\right)\right| \geq 4 \mu K^{-2}$.

Therefore

$$
\left|\operatorname{det}\left(N_{\gamma}\left(z_{1}\right), N_{\gamma}\left(z_{2}\right), \frac{d N_{\gamma}\left(z_{2}(t)\right)}{d t}\right)\right| \geq \mu K^{-2},
$$

and since the integrand in (38) has constant sign, we see that

$$
R^{-1 / 2+2 \delta} \geq\left|\int_{0}^{t_{2}} \mu K^{-2} d t\right|
$$

Hence, $\left|t_{2}\right| \leq K^{2} R^{-1 / 2+2 \delta}$ and, since the curve $t \mapsto z_{2}(t)$ is parametrized by arc length, we find that $\left|z_{2}-z_{2}^{\prime}\right| \lesssim K^{2} R^{-1 / 2+2 \delta}$. Since $z_{1}-z_{1}^{\prime}=z_{2}^{\prime}-z_{2}$ by (35), we also get $\left|z_{1}-z_{1}^{\prime}\right| \lesssim K^{2} R^{-1 / 2+2 \delta}$.

In a similar way, we see that $\left|z_{1}-z_{1}^{\prime}\right| \leq K^{2} R^{-1 / 2+2 \delta}$.

Hence, given $T_{1}$ and $T_{2}$, there are at most $R^{O(\delta)}$ possible tubes $T_{1}^{\prime}, T_{2}^{\prime}$ which give a non-negligible contribution to (34), and by Schur's lemma this implies that

$$
\int_{Q}\left|\mathcal{E}_{\gamma} f_{\tau_{1}, j, \text { tang }}\right|^{2}\left|\mathcal{E}_{\gamma} f_{\tau_{2}, j, \text { tang }}\right|^{2} \leq R^{O(\delta)} \sum_{T_{1}, T_{2} \in \mathbb{T}_{j, \text { tang, }}} \int\left|f_{\tau_{1}, T_{1}} d \sigma_{\gamma} * f_{\tau_{2}, T_{2}} d \sigma_{\gamma}\right|^{2}+\text { neglig. }
$$

Finally, note that Lemma 4.15 implies that $T_{1} \cap T_{2}$ is contained in a cube of side length $K R^{1 / 2+\delta}$. Hence, the same reasoning used to prove inequality (38) in [14] leads to

$$
\int\left|f_{\tau_{1}, T_{1}} d \sigma_{\gamma} * f_{\tau_{2}, T_{2}} d \sigma_{\gamma}\right|^{2} \leq R^{-1 / 2}\left\|f_{\tau_{1}, T_{1}}\right\|_{2}^{2}\left\|f_{\tau_{2}, T_{2}}\right\|_{2}^{2},
$$

and combining these two estimates we complete the proof of Lemma 4.14.

\subsection{Completing the proof of Theorem 3.2}

Following [14], pp. 396-398, we use induction on the size of $R$, the radius of $B_{R}$. Moreover, for given $R$, we also induct on the size of $\sum_{\tau} \int\left|f_{\tau}\right|^{2}$. Here we understand that a positive quantity is of size $2^{k}, k \in \mathbb{Z}$, if it lies in the interval $\left(2^{k-1}, 2^{k}\right]$.

Bases of induction. a) We recall from Remark 4.1 b) that for $1 \leq R \leq 1000 e^{e^{\epsilon^{-12}}}$

$$
\int_{B_{R}}\left|\mathcal{E}_{\gamma} f\right|^{3.25} \leq C_{4}(\epsilon)\left(\sum_{\tau}\left\|f_{\tau}\right\|_{2}^{2}\right)^{3 / 2+\epsilon}
$$

so that the estimate in Theorem 3.2 holds true for this range of $R$ 's. 
b) Also, if $\sum_{\tau} \int\left|f_{\tau}\right|^{2} \leq R^{-1000}$, then the estimate in Theorem 3.2 holds trivially, since

$$
\begin{aligned}
& \int_{B_{R}}\left|\mathcal{E}_{\gamma} f\right|^{3.25} \leq R^{3}\|f\|_{1}^{3.25} \leq R^{3}\|f\|_{2}^{3.25} \leq R^{-100}\|f\|_{2}^{3+2 \epsilon} \\
& \quad \leq K(\epsilon)^{2} R^{-100}\left(\sum_{\tau} \int\left|f_{\tau}\right|^{2}\right)^{3 / 2+\epsilon}
\end{aligned}
$$

In the induction procedure, it will thus suffice to show that in each step we can reduce to situations where either $R$, or $\sum_{\tau} \int\left|f_{\tau}\right|^{2}$, becomes smaller by a factor $\leq 1 / 2$, until we go below one of the thresholds described in a), or b).

We shall show that inequality (5) of Theorem 3.2 will then hold with the constant $C_{\epsilon}:=$ $\max \left\{K(\epsilon)^{2}, C_{4}(\epsilon)\right\}$.

Induction hypotheses. Assume that Theorem 3.2 holds for all radii $\leq R / 2$, or, given $R$, for all functions $g$ in place of $f$ such that $\sum_{\tau} \int\left|g_{\tau}\right|^{2} \leq \frac{1}{2} \sum_{\tau} \int\left|f_{\tau}\right|^{2}$ and every $\mu \geq 1$.

Write

$$
\int_{B_{R}}\left(B r_{\alpha} \mathcal{E}_{\gamma} f\right)^{3.25}=\sum_{i} \int_{B_{R} \cap O_{i}^{\prime}}\left(B r_{\alpha} \mathcal{E}_{\gamma} f\right)^{3.25}+\int_{B_{R} \cap W}\left(B r_{\alpha} \mathcal{E}_{\gamma} f\right)^{3.25}
$$

Case 1. Assume that the first term (cellular term) dominates (39). In this case, by (6) there will be $\sim D^{3}$ cells $O_{i}^{\prime}$, and for each of them

$$
\int_{B_{R} \cap O_{i}^{\prime}} B r_{\alpha} \mathcal{E}_{\gamma} f^{3.25} \sim D^{-3} \int_{B_{R}} B r_{\alpha} \mathcal{E}_{\gamma} f^{3.25} .
$$

In combination with Lemma 4.7, then, for every $i$,

$$
\begin{aligned}
\int_{B_{R}}\left(B r_{\alpha} \mathcal{E}_{\gamma} f\right)^{3.25} & \sim D^{3} \int_{B_{R} \cap O_{i}^{\prime}}\left(B r_{\alpha} \mathcal{E}_{\gamma} f\right)^{3.25} \\
& \lesssim D^{3} \int_{B_{R} \cap O_{i}^{\prime}}\left(B r_{2 \alpha} \mathcal{E}_{\gamma} f_{i}\right)^{3.25}+R^{-900}\left(\sum_{\tau}\left\|f_{\tau}\right\|_{2}\right)^{3.25}
\end{aligned}
$$

If the second term in (40) dominates, then, since $\alpha \geq K^{\epsilon}, R^{\delta_{\text {trans }} \log \left(K^{\epsilon} \alpha \mu\right)} \geq R^{\delta_{\text {trans }}} \geq 1$, and that finishes the proof.

If the first term in (40) dominates, we use Lemma 4.4 and the following immediate analogue to Lemma 2.7 in [14]:

Lemma 4.16 Consider some subsets $\mathbb{T}_{i} \subset \mathbb{T}$ indexed by $i \in \mathcal{I}$. If each tube $T$ belongs to at most $\kappa$ of the subsets $\left\{\mathbb{T}_{i}\right\}_{i \in \mathcal{I}}$, then, for every $\theta$,

$$
\sum_{i \in \mathcal{I}} \int_{3 \theta}\left|f_{\tau, i}\right|^{2} \leq \kappa \int_{10 \theta}\left|f_{\tau}\right|^{2}
$$

and

$$
\sum_{i \in \mathcal{I}} \int\left|f_{\tau, i}\right|^{2} \leq \kappa \int\left|f_{\tau}\right|^{2}
$$

Applying this lemma in combination with Lemma 4.4, we see that for each $\tau$,

$$
\sum_{i} \int\left|f_{\tau, i}\right|^{2} \leq(D+1) \int\left|f_{\tau}\right|^{2}
$$


and therefore

$$
\sum_{i} \sum_{\tau} \int\left|f_{\tau, i}\right|^{2} \leq(D+1) \sum_{\tau} \int\left|f_{\tau}\right|^{2} \text {. }
$$

Now, recall that there are $\sim D^{3}$ indices $i$. Thus we can choose and fix an index $i_{0}$ such that

$$
\sum_{\tau} \int\left|f_{\tau, i_{0}}\right|^{2} \lesssim D D^{-3} \sum_{\tau} \int\left|f_{\tau}\right|^{2}=D^{-2} \sum_{\tau} \int\left|f_{\tau}\right|^{2} \ll \frac{1}{2} \sum_{\tau} \int\left|f_{\tau}\right|^{2} .
$$

We finish this case by applying the induction hypothesis (on the size of $\sum_{\tau} \int\left|g_{\tau}\right|^{2}$ ) to the function $f_{i_{0}}:=\sum_{\tau} f_{\tau, i_{0}}$. Note that the support of $f_{\tau, i_{0}}$ is a tiny neighborhood of $\tau$. For this reason we need $\mu$ in the statement of Theorem 3.2, so that here we can apply the induction hypothesis with $2 \mu$ in place of $\mu$.

To this end, note also that

$$
\oint_{B\left(\omega, R^{-1 / 2}\right)}\left|f_{\tau, i_{0}}\right|^{2} \leq C \oint_{B\left(\omega, 10 R^{-1 / 2}\right)}\left|f_{\tau}\right|^{2} \leq C,
$$

where the first inequality is a consequence of the following immediate analogue to Lemma 2.8 in [14]:

Lemma 4.17 If $\mathbb{T}_{i} \subset \mathbb{T}$, then for any cap $\theta$, and any $\tau$,

$$
\int_{3 \theta}\left|f_{\tau, i}\right|^{2} \leq \int_{10 \theta}\left|f_{\tau}\right|^{2}
$$

We then apply our induction hypothesis to $\frac{1}{\sqrt{2 C}} f_{i_{0}}=\frac{1}{\sqrt{2 C}} \sum_{\tau} f_{\tau, i_{0}}$. Since we assume that the first term in (40) dominates, this yields

$$
\begin{aligned}
\int_{B_{R}}\left(B r_{\alpha} \mathcal{E}_{\gamma} f\right)^{3.25} & \lesssim D^{3} \int_{B_{R} \cap O_{i_{0}}^{\prime}}\left(B r_{2 \alpha} \mathcal{E}_{\gamma} f_{i_{0}}\right)^{3.25} \\
& \leq(2 C)^{1 / 8-\epsilon} D^{3} C_{\epsilon} R^{\epsilon}\left(\sum_{\tau} \int\left|f_{\tau, i_{0}}\right|^{2}\right)^{3 / 2+\epsilon} R^{\delta_{\text {trans }} \log \left(K^{\epsilon} 2 \alpha 2 \mu\right)},
\end{aligned}
$$

and thus by (41)

$$
\begin{aligned}
\int_{B_{R}}\left(B r_{\alpha} \mathcal{E}_{\gamma} f\right)^{3.25} & \leq C_{1} D^{3} C_{\epsilon} R^{\epsilon}\left(D^{-2} \sum_{\tau} \int\left|f_{\tau}\right|^{2}\right)^{3 / 2+\epsilon} R^{\delta_{\text {trans }} \log \left(K^{\epsilon} \alpha \mu\right)} R^{c \delta_{\text {trans }}} \\
& =C_{1} C_{\epsilon} R^{\epsilon}\left(\sum_{\tau} \int\left|f_{\tau}\right|^{2}\right)^{3 / 2+\epsilon} R^{\delta_{\text {trans }} \log \left(K^{\epsilon} \alpha \mu\right)} D^{-2 \epsilon} R^{c \delta_{\text {trans }}} \\
& \leq C_{\epsilon} R^{\epsilon}\left(\sum_{\tau} \int\left|f_{\tau}\right|^{2}\right)^{3 / 2+\epsilon} R^{\delta_{\text {trans }} \log \left(K^{\epsilon} \alpha \mu\right)},
\end{aligned}
$$

closing the induction.

Case 2. Assume that the second term (wall term) dominates (39). In this case we apply Lemma 4.9 to obtain 


$$
\begin{aligned}
\int_{B_{R}}\left(B r_{\alpha} \mathcal{E}_{\gamma} f\right)^{3.25} \leq & C_{\epsilon} \sum_{j} \int_{B_{j} \cap W} \sum_{I}\left(B r_{60 \alpha} \mathcal{E}_{\gamma} f_{I, j, \text { trans }}\right)^{3.25} \\
& +C K^{325} \sum_{j} \int_{B_{j} \cap W} \operatorname{Bil}\left(\mathcal{E}_{\gamma} f_{j, \text { tang }}\right)^{3.25}+C\left(R^{-900} \sum_{\tau}\left\|f_{\tau}\right\|_{2}\right)^{3.25}
\end{aligned}
$$

(note that the number of all possible subsets I of the given family of caps is only a constant depending on $\epsilon$ ).

Again, if the third term of this last sum dominates, the proof is easily finished.

If the second term dominates, then by Proposition 4.13, since $K \ll R$,

$$
\begin{aligned}
\int_{B_{R}}\left(B r_{\alpha} \mathcal{E}_{\gamma} f\right)^{3.25} & \lesssim C_{\epsilon} K^{325} \sum_{j} \int_{B_{j} \cap W} \operatorname{Bil}\left(\mathcal{E}_{\gamma} f_{j, \text { tang }}\right)^{3.25} \\
& \leq C_{\epsilon} K^{325} R^{O(\delta)+\epsilon / 2}\left(\sum_{\tau} \int\left|f_{\tau}\right|^{2}\right)^{3 / 2+\epsilon} \leq C R^{\epsilon}\left(\sum_{\tau} \int\left|f_{\tau}\right|^{2}\right)^{3 / 2+\epsilon} .
\end{aligned}
$$

This finishes the proof in this case.

Finally, assume that the first term in (42) dominates. Then, since the ball $B_{j}$ has radius $R^{1-\delta}<\frac{R}{2}$, we shall induct on the size of $R$. Note also that $f_{\tau, j, \text { trans }, I}$ is supported in a tiny neighborhood of $\tau$, so we shall again apply the induction hypothesis with $2 \mu$ in place of $\mu$.

By Lemma 4.17,

$$
\oint_{B\left(\omega, R^{-1 / 2}\right)}\left|f_{I, j, t \text { trans }, \tau}\right|^{2} \leq \oint_{B\left(\omega, R^{-1 / 2}\right)}\left|f_{\tau}\right|^{2} \leq C,
$$

which implies the same kind of control over larger balls of radius $\left(R^{1-\delta}\right)^{-1 / 2}$. Thus, $\frac{1}{C} f_{I, j, t r a n s}$ satisfies the induction hypothesis of Theorem 3.2, and therefore

$$
\int_{B_{j} \cap W}\left(B r_{60 \alpha} \mathcal{E}_{\gamma} f_{I, j, \text { trans }}\right)^{3.25} \leq C_{\epsilon} R^{\epsilon(1-\delta)}\left(\sum_{\tau \in I} \int\left|f_{\tau, j, \text { trans }}\right|^{2}\right)^{3 / 2+\epsilon} R^{\delta_{\text {trans }}(1-\delta) \log \left(60 K^{\epsilon} \alpha \mu\right)} .
$$

By Lemma 4.5,

$$
\sum_{j} \int\left|f_{\tau, j, \text { trans }}\right|^{2} \leq \operatorname{Poly}(D) \int\left|f_{\tau}\right|^{2}
$$

Moreover,

$$
\sum_{I, j}\left(\sum_{\tau \in I} \int\left|f_{\tau, j, \text { trans }}\right|^{2}\right)^{3 / 2+\epsilon} \leq \sum_{I}\left(\sum_{j} \sum_{\tau \in I} \int\left|f_{\tau, j, \text { trans }}\right|^{2}\right)^{3 / 2+\epsilon} .
$$

Since there are at most $M_{\epsilon}$ families $I$, combining these estimates we see that

$$
\sum_{I, j}\left(\sum_{\tau \in I} \int\left|f_{\tau, j, t \text { trans }}\right|^{2}\right)^{3 / 2+\epsilon} \leq M_{\epsilon} \operatorname{Poly}(D)\left(\sum_{\tau} \int\left|f_{\tau}\right|^{2}\right)^{3 / 2+\epsilon},
$$


and thus finally

$$
\begin{aligned}
\int_{B_{R}}\left(B r_{\alpha} \mathcal{E}_{\gamma} f\right)^{3.25} & \leq M_{\epsilon} C_{\epsilon} \operatorname{Poly}(D) R^{\epsilon(1-\delta)}\left(\sum_{\tau} \int\left|f_{\tau}\right|^{2}\right)^{3 / 2+\epsilon} R^{\delta_{\text {trans }}(1-\delta) \log \left(60 K^{\epsilon} \alpha \mu\right)} \\
& \leq\left(M_{\epsilon} \operatorname{Poly}(D) R^{-\delta \epsilon+c \delta_{\text {trans }}}\right) C_{\epsilon} R^{\epsilon}\left(\sum_{\tau} \int\left|f_{\tau}\right|^{2}\right)^{3 / 2+\epsilon} R^{\delta_{\text {trans }} \log \left(K^{\epsilon} \alpha \mu\right)}
\end{aligned}
$$

By our choices of $\delta$ and $\delta_{\text {trans }}$, since we assume that $R$ is sufficiently large, we find that the first factor in parentheses is bounded by 1 , and thus

$$
\int_{B_{R}}\left(B r_{\alpha} \mathcal{E}_{\gamma} f\right)^{3.25} \leq C_{\epsilon} R^{\epsilon}\left(\sum_{\tau} \int\left|f_{\tau}\right|^{2}\right)^{3 / 2+\epsilon} R^{\delta_{\text {trans }} \log \left(K^{\epsilon} \alpha \mu\right)} .
$$

This closes the induction and thus completes the proof of Theorem 3.2.

\subsection{Proof of the Geometric Lemma}

In this subsection we prove Lemma 4.11. Assume that we are given a family of caps $\left\{\tau_{k}\right\}$ such that for any $k, m$ with $k \neq m$ we have

$$
\min \left\{\left|y_{m}^{c}-y_{k}^{c}\right|, \max \left\{\left|\mathbf{t}_{z_{m}^{c}}^{\gamma}\left(z_{m}^{c}, z_{k}^{c}\right)\right|,\left|\mathbf{t}_{z_{k}^{c}}^{\gamma}\left(z_{m}^{c}, z_{k}^{c}\right)\right|\right\}\right\} \leq 10 \mu^{1 / 2} K^{-1},
$$

where we denote by $z_{k}^{c}=\left(x_{k}^{c}, y_{k}^{c}\right)$ the center of the cap $\tau_{k}$.

Case 1. For all $k, m$ we have $\left|y_{m}^{c}-y_{k}^{c}\right| \leq 10 \mu^{1 / 2} K^{-1}$. Then, all caps are contained in a horizontal strip of width $10 \mu^{1 / 2} K^{-1} \leq \mu^{1 / 2} K^{-1 / 4}$, since $K \geq 30$.

Case 2. There are two caps, say $\tau_{1}, \tau_{2}$, such that $\left|y_{1}^{c}-y_{2}^{c}\right|>10 \mu^{1 / 2} K^{-1}$. We may assume that $y_{2}^{c}-y_{1}^{c}=\max _{j \neq k}\left|y_{j}^{c}-y_{k}^{c}\right|$. Then, for all $k$,

$$
y_{1}^{c} \leq y_{k}^{c} \leq y_{2}^{c} .
$$

Since $\tau_{1}$ and $\tau_{2}$ are not strongly separated, $\left|\mathbf{t}_{z_{1}^{c}}^{\gamma}\left(z_{1}^{c}, z_{2}^{c}\right)\right| \leq 10 \mu^{1 / 2} K^{-1}$ and $\left|\mathbf{t}_{z_{2}^{c}}^{\gamma}\left(z_{1}^{c}, z_{2}^{c}\right)\right| \leq$ $10 \mu^{1 / 2} K^{-1}$. Therefore, by (18),

$$
|\gamma|\left|y_{2}^{c}-y_{1}^{c}\right|^{2}=\left|\mathbf{t}_{z_{1}^{c}}^{\gamma}\left(z_{1}^{c}, z_{2}^{c}\right)-\mathbf{t}_{z_{2}^{c}}^{\gamma}\left(z_{1}^{c}, z_{2}^{c}\right)\right| \leq 20 \mu^{1 / 2} K^{-1},
$$

and since $\left|y_{k}^{c}-y_{1}^{c}\right| \leq\left|y_{2}^{c}-y_{1}^{c}\right|$, we see that for all $k$

$$
\left|y_{k}^{c}-y_{1}^{c}\right| \leq\left(20 \mu^{1 / 2}|\gamma|^{-1} K^{-1}\right)^{\frac{1}{2}} .
$$

In combination with (18), this also implies that

$$
\begin{aligned}
\left|x_{k}^{c}-x_{1}^{c}\right| & \leq\left|\mathbf{t}_{z_{1}^{c}}^{\gamma}\left(z_{1}^{c}, z_{k}^{c}\right)\right|+|\gamma|\left|y_{k}^{c}\right|\left|y_{k}^{c}-y_{1}^{c}\right| \\
& \leq 10 \mu^{1 / 2} K^{-1}+|\gamma|\left(20 \mu^{1 / 2}|\gamma|^{-1} K^{-1}\right)^{\frac{1}{2}} \\
& \leq 15 \mu^{1 / 2} K^{-1 / 2},
\end{aligned}
$$

since $|\gamma| \leq 1, \mu \geq 1$ and $K \geq 1$.

When $|\gamma| K^{1 / 2}>1$, we conclude from (44) that $\left|y_{k}^{c}-y_{1}^{c}\right| \leq\left(20 \mu^{1 / 2} K^{-1 / 2}\right)^{1 / 2}$ for all $k$. Hence, all the caps are contained in a horizontal strip of width $10 \mu^{1 / 2} K^{-1 / 4}$. Decomposing these further into horizontal strips of width $0.5 \mu^{1 / 2} K^{-1 / 4}$, each of which is contained in one of the ragged strips $S_{\ell}$ that have been fixed in Section 3, and distributing the caps $\tau_{k}$ of our family over these ragged strips, we arrive at at most 40 horizontal ragged strips of 
width $\mu^{1 / 2} K^{-1 / 4}$ which contain all the caps considered in Case 2 . Note that by our passage to ragged strips the width does not increase by more than $2 \mu^{1 / 2} K^{-1} \leq 0.5 \mu^{1 / 2} K^{-1 / 4}$, since $K \geq 20$.

When $|\gamma| K^{1 / 2} \leq 1$, we conclude from (45) that all the caps are contained in a vertical strip of width $20 \mu^{\overline{1} / 2} K^{-1 / 2}$, and arguing as before we can conclude the proof of Lemma 4.11 also in this case.

\section{Passing from extension estimates on cubes to estimates on plates: an orthogonality lemma}

We will here finally discuss an auxilary lemma that we needed in Section 2.

Let $\Omega \subset \mathbb{R}^{n}$ be an open bounded set and $\phi: \Omega \rightarrow \mathbb{R}$ any phase function such that $|\nabla \phi(x)| \lesssim 1$ for all $x \in \Omega$. Assume further that $\rho \in C^{\infty}(\Omega)$, and consider the Fourier extension operator

$$
\mathcal{E} f(\xi):=\int_{\Omega} f(x) e^{-i\left[\xi^{\prime} \cdot x+\xi_{n+1} \phi(x)\right]} \rho(x) d x,
$$

where $\xi=\left(\xi^{\prime}, \xi_{n+1}\right) \in \mathbb{R}^{n} \times \mathbb{R}$ (for convenience, we have chosen here a different sign in the phase than in the definition of $\mathcal{E}_{\gamma} f$ ).

Lemma 5.1 Let $2 \leq q \leq p$. Assume that for every $\epsilon>0$ there exists a constant $C_{\epsilon}$ such that for every $R \geq 1$

$$
\|\mathcal{E} f\|_{L^{p}\left([0, R]^{n+1}\right)} \leq C_{\epsilon} R^{\epsilon}\|f\|_{L^{2}(\Omega)}^{2 / q}\|f\|_{L^{\infty}(\Omega)}^{1-2 / q}
$$

for all $f \in L^{q}(\Omega)$. Then for every $\epsilon>0$ there exists a constant $C_{\epsilon}^{\prime}$ such that for all $R \geq 1$

$$
\|\mathcal{E} f\|_{L^{p}\left(\mathbb{R}^{n} \times[0, R]\right)} \leq C_{\epsilon}^{\prime} R^{\epsilon}\|f\|_{L^{2}(\Omega)}^{2 / q}\|f\|_{L^{\infty}(\Omega)}^{1-2 / q}
$$

for all $f \in L^{q}(\Omega)$.

Proof First observe that (46) holds for any translate of $[0, R]^{n+1}$ in place of $[0, R]^{n+1}$ as well, in particular on any cube $Q_{y}:=R(y, 0)+[0, R]^{n+1}, y \in \mathbb{Z}^{n}$.

In order to pass to a corresponding estimate on the plate $\left.\mathbb{R}^{n} \times[0, R]\right)$, which decomposes into the cubes $Q_{y}$, it will suffice to perform an adapted frequency decomposition of $f$ (a full wave packet decomposition is needed here):

Let $f_{y}:=f * \check{\chi}_{y}$, where $\chi_{y}(\eta):=\chi(\eta / R-y), y \in \mathbb{Z}^{n}$, and $\chi$ is a suitable compactly supported bump function chosen so that the $\chi_{y}, y \in \mathbb{Z}^{n}$, form a partition of unity on $\mathbb{R}^{n}$. Then $f=\sum_{y} f_{y}$.

In order to prove (47), we may and shall assume that $0 \leq \xi_{n+1} \leq R$. Under this restriction, we will see that $\mathcal{E} f_{y}$ is essentially supported in $Q_{y}$. Indeed, note that by Fourier inversion

$$
\mathcal{E} f_{y}(\xi)=c_{n} \iint \hat{f}(\eta) \chi(\eta / R-y) e^{-i\left[\left(\xi^{\prime}-\eta\right) \cdot x+\xi_{n+1} \phi(x)\right]} \rho(x) d x d \eta
$$

The gradient in $x$ of the full phase is given by $\xi^{\prime}-\eta+\xi_{n+1} \nabla \phi(x)=\xi^{\prime}-R y+O(R)$. Hence, by a standard integration by parts argument in $x$, we see that for any $N \in \mathbb{N}$

$$
\left|\mathcal{E} f_{y}(\xi)\right| \lesssim_{N} R^{n}\left|\xi^{\prime}-R y\right|^{-N}\left\|f_{y}\right\|_{1}, \quad \text { if }\left|\xi^{\prime}-R y\right| \gg R .
$$


It is thus natural to split (and estimate, using Minkowski's inequality)

$$
\|\mathcal{E} f\|_{L^{p}\left(\mathbb{R}^{n} \times[0, R]\right)}=\left(\sum_{y}\left\|\sum_{z} \mathcal{E} f_{y+z}\right\|_{L^{p}\left(Q_{y}\right)}^{p}\right)^{1 / p} \leq \sum_{z}\left(\sum_{y}\left\|\mathcal{E} f_{y}\right\|_{L^{p}\left(Q_{y-z}\right)}^{p}\right)^{1 / p}
$$

into two parts: First we use (46) and $p \geq q$ to estimate

$$
\begin{aligned}
\sum_{|z| \lesssim 1}\left(\sum_{y}\left\|\mathcal{E} f_{y}\right\|_{L^{p}\left(Q_{y-z}\right)}^{p}\right)^{1 / p} & \lesssim C_{\epsilon} R^{\epsilon}\left(\sum_{y}\left\|f_{y}\right\|_{L^{2}(\Omega)}^{2 p / q}\left\|f_{y}\right\|_{L^{\infty}(\Omega)}^{p(1-2 / q)}\right)^{1 / p} \\
& \leq C_{\epsilon} R^{\epsilon}\left(\sum_{y}\left\|f_{y}\right\|_{L^{2}(\Omega)}^{2}\right)^{1 / q}\|f\|_{L^{\infty}(\Omega)}^{1-2 / q} \\
& \lesssim C_{\epsilon} R^{\epsilon}\|f\|_{L^{2}(\Omega)}^{2 / q}\|f\|_{L^{\infty}(\Omega)}^{1-2 / q},
\end{aligned}
$$

where have used Plancherel's theorem. The remainder can be estimated using (48):

$$
\begin{aligned}
\sum_{|z| \gg 1}\left(\sum_{y}\left\|\mathcal{E} f_{y}\right\|_{L^{p}\left(Q_{y-z}\right)}^{p}\right)^{1 / p} & \lesssim R^{n+(n+1) / p} \sum_{|z| \gg 1}(R|z|)^{-N}\left(\sum_{y}\left\|f_{y}\right\|_{1}^{p}\right)^{1 / p} \\
& \lesssim R^{-N^{\prime}}\left(\sum_{y}\left\|f_{y}\right\|_{1}^{p}\right)^{1 / p}
\end{aligned}
$$

which finishes the proof because $\left\|f_{y}\right\|_{1} \leq\left\|f_{y}\right\|_{2}^{2 / q}\left\|f_{y}\right\|_{\infty}^{1-2 / q}$, so that from here we can proceed as before.

Funding Open Access funding enabled and organized by Projekt DEAL.

Open Access This article is licensed under a Creative Commons Attribution 4.0 International License, which permits use, sharing, adaptation, distribution and reproduction in any medium or format, as long as you give appropriate credit to the original author(s) and the source, provide a link to the Creative Commons licence, and indicate if changes were made. The images or other third party material in this article are included in the article's Creative Commons licence, unless indicated otherwise in a credit line to the material. If material is not included in the article's Creative Commons licence and your intended use is not permitted by statutory regulation or exceeds the permitted use, you will need to obtain permission directly from the copyright holder. To view a copy of this licence, visit http://creativecommons.org/licenses/by/4.0/.

\section{References}

1. Barron, A.: Restriction estimates for hyperboloids in higher dimensions via bilinear estimates. Revista Mat. Iberoam. (2021) (Online first), https://doi.org/10.4171/RMI/1310.

2. Bejenaru, I.: Optimal bilinear restriction estimates for general hypersurfaces and the role of the shape operator. Int. Math. Res. Not. IMRN 23, 7109-7147 (2017)

3. Bennet, J., Carbery, A., Tao, T.: On the multilinear restriction and Kakeya conjectures. Acta Math. 196(2), 261-302 (2006)

4. Bourgain, J.: Besicovitch-type maximal operators and applications to Fourier analysis. Geom. Funct. Anal. 22, 147-187 (1991)

5. Bourgain, J.: Some new estimates on oscillatory integrals. Essays in Fourier Analysis in honor of E. M. Stein. Princeton Math. Ser. 42, Princeton University Press, Princeton, NJ, 83-112 (1995)

6. Bourgain, J.: Estimates for cone multipliers. Oper. Theory Adv. Appl. 77, 1-16 (1995)

7. Bourgain, J., Guth, L.: Bounds on oscillatory integral operators based on multilinear estimates. Geom. Funct. Anal. 21, 1239-1295 (2011) 
8. Buschenhenke, S., Müller, D., Vargas, A.: A Fourier restriction theorem for a two-dimensional surface of finite type. Anal. PDE 10(4), 817-893 (2017)

9. Buschenhenke, S., Müller, D., Vargas, A.: A Fourier restriction theorem for a perturbed hyperbolic paraboloid. Proc. Lond. Math. Soc. (3) 120, 124-154 (2020)

10. Buschenhenke, S., Müller, D., Vargas, A.: On Fourier restriction for finite-type perturbations of the hyperbolic paraboloid. Springer INdAM Series (Conference Proceedings) Vol. 45, pp. 193-222; ISBN 978-3-030-72057-5, ISBN 978-3-030-72058-2 (eBook) (2021)

11. Buschenhenke, S., Müller, D., Vargas, A.: Partitions of flat one-variate functions and a Fourier restriction theorem for related perturbations of the hyperbolic paraboloid. J. Geom. Anal. 31(7), 6941-6986 (2021)

12. Cho, C.-H., Lee, J.: Improved restriction estimate for hyperbolic surfaces in $\mathbb{R}^{3}$. J. Funct. Anal. 273(3), 917-945 (2017)

13. Fefferman, C.: Inequalities for strongly singular convolution operators. Acta Math. 9-36 (1970)

14. Guth, L.: A restriction estimate using polynomial partitioning. J. Am. Math. Soc. 29(2), 371-413 (2016)

15. Guth, L.: Restriction estimates using polynomial partitioning II. Acta Math. 221(1), 81-142 (2016)

16. Greenleaf, A.: Principal Curvature and Harmonic Analysis. Indiana Univ. Math. J. 30, No. 4 (1981)

17. Hickman, J., Rogers, K.: Improved Fourier restriction estimates in higher dimensions. Camb. J. Math. 7(3), 219-282 (2019)

18. Ikromov, I.A., Kempe, M., Müller, D.: Estimates for maximal functions associated with hypersurfaces in $\mathbb{R}^{3}$ and related problems in harmonic analysis. Acta Math. 204, 151-271 (2010)

19. Ikromov, I.A., Müller, D.: Uniform estimates for the Fourier transform of surface carried measures in $\mathbb{R}^{3}$ and an application to Fourier restriction. J. Fourier Anal. Appl. 17(6), 1292-1332 (2011)

20. Ikromov, I. A., Müller, D.: Fourier restriction for hypersurfaces in three dimensions and Newton polyhedra. Annals of Mathematics Studies, 194. Princeton University Press, Princeton, NJ (2016)

21. Kim, J.: Some remarks on Fourier restriction estimates, preprint 2017; available at arXiv:1702.01231

22. Lee, S.: Bilinear restriction estimates for surfaces with curvatures of different signs. Trans. Am. Math. Soc. 358(8), 3511-2533 (2005)

23. Lee, S., Vargas, A.: Restriction estimates for some surfaces with vanishing curvatures. J. Funct. Anal. 258(9), 2884-2909 (2010)

24. Moyua, A., Vargas, A., Vega, L.: Schrödinger maximal function and restriction properties of the Fourier transform. Internat. Math. Res. Notices 16, 793-815 (1996)

25. Moyua, A., Vargas, A., Vega, L.: Restriction theorems and maximal operators related to oscillatory integrals in $\mathbb{R}^{3}$. Duke Math. J. 96(3), 547-574 (1999)

26. Shayya, B.: Weighted restriction estimates using polynomial partitioning. Proc. Lond. Math. Soc. (3) 115(3), 545-598 (2017)

27. Stein, E.M.: Oscillatory Integrals in Fourier Analysis. Beijing Lectures in Harmonic Analysis. Princeton University Press, Princeton (1986)

28. Stovall, B.: Scale invariant Fourier restriction to a hyperbolic surface. Anal. PDE 12(5), 1215-1224 (2019)

29. Strichartz, R.S.: Restrictions of Fourier transforms to quadratic surfaces and decay of solutions of wave equations. Duke Math. J. 44(3), 705-714 (1977)

30. Tao, T.: A Sharp bilinear restriction estimate for paraboloids. Geom. Funct. Anal. 13, 1359-1384 (2003)

31. Tao, T., Vargas, A.: A bilinear approach to cone multipliers I. Restriction estimates. Geom. Funct. Anal. 10, 185-215 (2000)

32. Tao, T., Vargas, A.: A bilinear approach to cone multipliers II. Applications. Geom. Funct. Anal. 10, 216-258 (2000)

33. Tao, T., Vargas, A., Vega, L.: A bilinear approach to the restriction and Kakeya conjectures. J. Am. Math. Soc. 11(4), 967-1000 (1998)

34. Tomas, P.A.: A restriction theorem for the Fourier transform. Bull. Am. Math. Soc. 81, 477-478 (1975)

35. Vargas, A.: Restriction theorems for a surface with negative curvature. Math. Z. 249, 97-111 (2005)

36. Wang, Hong, A restriction estimate in $\mathbb{R}^{3}$ using brooms. To appear Duke Math. J Available at arXiv: 1802.04312

37. Wolff, T.: A sharp bilinear cone restriction estimate. Ann. Math., Second Series, Vol. 153, no. 3, 661-698 (2001)

38. Zygmund, A.: On Fourier coefficients and transforms of functions of two variables. Stud. Math. 50, 189-201 (1974)

Publisher's Note Springer Nature remains neutral with regard to jurisdictional claims in published maps and institutional affiliations. 